\title{
LINEAR POWER-EFFICIENT RF AMPLIFIER WITH PARTIAL POSITIVE FEEDBACK
}

\author{
A Thesis \\ presented to \\ the Faculty of California Polytechnic State University, \\ San Luis Obispo
}

\author{
In Partial Fulfillment \\ of the Requirements for the Degree \\ Master of Science in Electrical Engineering
}

by

Matthew E. King

June 2012 
(C) 2012

Matthew E. King

ALL RIGHTS RESERVED 


\section{COMMITTEE MEMBERSHIP}

TITLE:

Linear Power-Efficient RF Amplifier with Partial

Positive Feedback

AUTHOR:

Matthew E. King

DATE SUBMITTED:

June 2012

COMMITTEE CHAIR:

Vladimir I. Prodanov, Ph.D., Assistant Professor, Electrical Engineering

COMMITTEE MEMBER:

Dennis Derickson, Ph.D., Associate Professor,

Electrical Engineering

COMMITTEE MEMBER:

Tina Smilkstein, Ph.D., Assistant Professor,

Electrical Engineering 


\section{ABSTRACT \\ Linear Power-Efficient RF Amplifier with Partial Positive Feedback \\ Matthew E. King}

Over the last decade, the number of mobile wireless devices on the market has increased substantially. New "multi-carrier" modulation schemes, such as OFDM, WCDMA, and WiMAX, have been developed to accommodate the increasing number of wireless subscribers and the demand for faster data rates within the limited commercial frequency spectrum. These complex modulation schemes create signals with high peak-to-average power ratios (PAPR), exhibiting rapid changes in the signal magnitude. To accommodate these high-PAPR signals, RF power amplifiers in mobile devices must operate under backed-off gain conditions, resulting in poor power efficiency. Various efficiency-enhancement solutions have been realized for backed-off devices to combat this issue.

A brief overview of one of the more extensively researched solutions, the Doherty amplifier, is given, and its inherent limitations are discussed. A recently proposed amplifier topology that provides the efficiency benefits of the Doherty amplifier, while overcoming some of the fundamental problems that plague the standard Doherty architecture, is investigated. A step-by-step design methodology is presented and confirmed by extensive simulation in Agilent ADS. A design example, tuned for maximum efficiency at peak output power, is implemented on a PCB and tested to verify the validity of the proposed circuit configuration. 


\section{ACKNOWLEDGMENTS}

I would first like to thank Dr. Vladimir Prodanov for the opportunity to work on such a fascinating and challenging project. Without his expertise in the field of amplifier design and his patience and willingness to spend long hours in the laboratory with me, the completion of this project would not have been possible.

I would also like to thank Dr. Dennis Derickson and Dr. Tina Smilkstein for sitting on my thesis committee, and for their thoughts and suggestions on the work.

Finally, I owe my parents, Robert and Jody, a debt of gratitude for their continued support and encouragement throughout the years, and enabling me to successfully complete the program here at Cal Poly. 


\section{Contents}

List of Tables $\quad$ viii

List of Figures $\quad$ xi

1 Introduction 1

1.1 Motivation . . . . . . . . . . . . . . . . . . . . . . 1

1.1 .1 Orthogonal Frequency Division Multiplexing (OFDM) . . . . . . . . . . 1

1.1 .2 The Linearity-Efficiency Tradeoff in PA Design . . . . . . . . . . . . . . 3

1.2 Power Amplifier Classes of Operation $\ldots \ldots \ldots \ldots$. . . . . . . . . . . . 4

1.3 The Doherty Amplifier $\ldots \ldots \ldots \ldots \ldots \ldots$

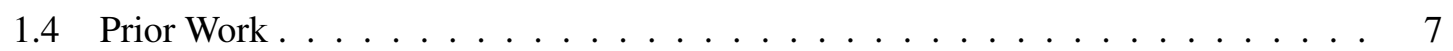

\begin{tabular}{|lll}
2 & The Negative Conductance Topology & 8
\end{tabular}

2.1 General Overview . . . . . . . . . . . . . . . . . . . . . . 8

2.2 The Impedance Inverter $\ldots \ldots \ldots \ldots$

2.3 Achieving the "Doherty Effect" via Transformer Feedback . . . . . . . . . . . . 11

$\begin{array}{lll}3 & \text { Design Methodology } & 14\end{array}$

$3.1 \quad$ Active Device Sizing $\ldots \ldots \ldots \ldots \ldots$

3.2 Impedance Inverter Sizing $\ldots \ldots \ldots \ldots \ldots$

3.3 Bias Circuit . . . . . . . . . . . . . . . . . . . . . . . . . . . . . . . 19

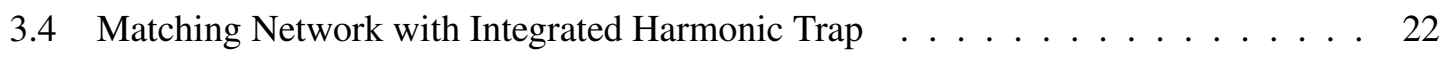


3.5 Class C Transformer Feedback . . . . . . . . . . . . . . . . . . . . . . . . . . . 24

3.6 Summary of Design Procedures $\ldots \ldots \ldots \ldots \ldots \ldots$

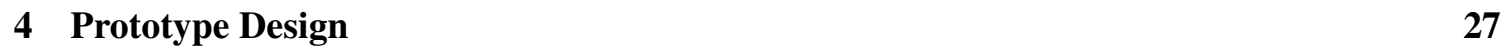

$4.1 \quad$ Class AB Stage $\ldots \ldots \ldots \ldots \ldots \ldots$

4.2 Impedance Inverter $\ldots \ldots \ldots \ldots$. . . . . . . . . . . . . . . . . . . 29

4.3 Class C Biasing and Sizing . . . . . . . . . . . . . . . . . . . . . . . . . . . . . 29

4.4 Matching Network and Harmonic Trap . . . . . . . . . . . . . . . . . . . . 31

4.5 Class C Feedback . . . . . . . . . . . . . . . . . . . . . . 34

4.6 Completed Amplifier $\ldots \ldots \ldots \ldots \ldots \ldots \ldots$

4.7 PCB Layout $\ldots \ldots \ldots \ldots$

5 Prototype Test Results 39

$5.1 \quad$ Prototype $1(\mathrm{PCB})$

5.2 Prototype 2 (Solderable Breadboard) $\ldots \ldots \ldots \ldots$. . . . . . . . . . . 42

6 Conclusions and Future Work $\quad 48$

\begin{tabular}{|ll|}
\hline Appendix A Circuit Schematics & 51
\end{tabular}

\begin{tabular}{|lll}
\hline Appendix B & PCB Layout Detail & 53
\end{tabular}

\begin{tabular}{ll}
\hline References & 55
\end{tabular} 


\section{List of Tables}

1.1 Conduction angles and maximum theoretical efficiencies for Class A, B, and C amplifiers $\ldots \ldots \ldots \ldots \ldots \ldots \ldots \ldots \ldots \ldots \ldots \ldots \ldots \ldots \ldots \ldots \ldots \ldots \ldots \ldots$

\begin{tabular}{|ll|l|l|}
\hline 3.1 & List of components in the proposed bias circuit of Figure & 3.3 & and their respective \\
\hline
\end{tabular}

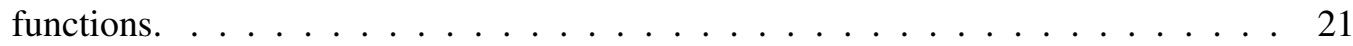

$4.1 \quad$ List of component values for the implemented bias circuit (Refer to Figure 3.3 and Table|3.1p. . . . . . . . . . . . . . . . . . . 31

4.2 Impedance and Q-factor at each labeled node in Figure 4.5 and Figure 4.6 . . . . . . 32

5.1 Comparison of theoretical and measured impedance inverter voltages. . . . . . . . 41

5.2 Comparison of simulated and measured DC bias levels. . . . . . . . . . . . . 43 


\section{List of Figures}

1.1 Summing of signals with varied amplitude and phase to form a high-PAPR signal.

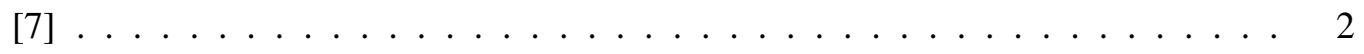

1.2 Time-domain waveform of an LTE signal. [6] . . . . . . . . . . . . . 2

1.3 Typical PAE curve for conventional Class AB amplifier. [7] . . . . . . . . . . . 3

1.4 Definition of conduction angle for Class A, B, and C operation. [9] . . . . . . . . 5

1.5 Voltage and current waveforms for Class A, B, and C operation. [9] . . . . . . . 5

1.6 Block diagram of Doherty amplifier. $\ldots \ldots \ldots \ldots \ldots$

2.1 Conceptual diagram of amplifier in negative conductance configuration. . . . . . . 8

2.2 Lumped element (LC) impedance inverter. . . . . . . . . . . . . . . . . . 9

2.3 Impedance inverter, represented as generic two-port network, connected to the Class AB amplifier (Port 1) and Class C amplifier (Port 2). . . . . . . . . . . . . . 10

2.4 Class C partial positive feedback configuration. An inverting transformer is connected in series with the output inductor of the impedance inverter. . . . . . . . . . 12

3.1 Ideal Class $\mathrm{AB}$ and Class $\mathrm{C}$ base voltages of arbitrary frequency, swept over a range of amplitudes. The maximum magnitudes of the Class AB and Class $\mathrm{C}$ base voltages are equal. . . . . . . . . . . . . . . . . . . 17

3.2 Simulation plot of DC component of Class AB and Class $\mathrm{C}$ base currents for the design presented in Chapter 4 . . . . . . . . . . . . . . . . . . 20

3.3 Proposed bias circuit. . . . . . . . . . . . . . . . . . . . 20

$3.4 \quad$ Base topology for Pi-section matching network. . . . . . . . . . . . . . 22 
3.5 Pi-section matching network with integrated harmonic trap . . . . . . . . . . . 23

3.6 Class C partial positive feedback configuration. . . . . . . . . . . . . . . . 24

4.1 Magnitude of Class AB collector current (fundamental component) vs. input voltage. 28

4.2 Class AB collector current (time-domain) for input voltages swept from $0 \mathrm{mV}$ to

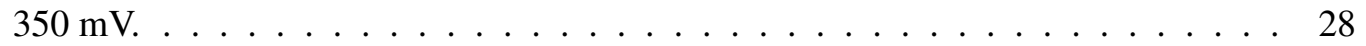

4.3 Ideal equation-based driving source for Class C stage in Agilent ADS. . . . . . . . 30

$4.4 \quad$ Results of idealized simulation to determine correct Class C biasing and device sizing. . . . . . . . . . . . . . . . . . 30

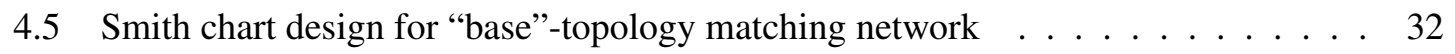

$4.6 \quad$ Matching network with resulting component values and node annotations. . . . . . 33

4.7 Completed matching network with harmonic trap. . . . . . . . . . . . . 33

4.8 Class C partial positive feedback configuration with calculated component values. . 34

4.9 Class C collector current and base voltage. . . . . . . . . . . . . . . . 34

4.10 Magnitudes of Class AB and Class C collector voltage (fundamental components) vs. input voltage. . . . . . . . . . . . . . . . . . . . 35

4.11 Magnitudes of Class AB and Class C collector current (fundamental components) vs. input voltage. . . . . . . . . . . . . . . . . . . 35

4.12 Peak amplitudes of Class AB and Class C collector voltage vs. input voltage. . . . 36

4.13 Peak amplitudes of output voltage vs. input voltage. . . . . . . . . . . . . 37

4.14 Power efficiency vs. input voltage. . . . . . . . . . . . . . . . . . 37

4.15 PCB layout of negative conductance amplifier. . . . . . . . . . . . . . 38

5.1 Completed PCB prototype. . . . . . . . . . . . . . . . . . . . 39

5.2 Voltage waveforms at impedance inverter input (Class AB side) and output (Class C side) . . . . . . . . . . . . . . . . . . . 40

5.3 Impedance inverter output voltage and Class $\mathrm{C}$ base voltage, verifying proper attenuation and inversion of the feedback signal. . . . . . . . . . . . . . . . . 41

5.4 Class C collector voltage and base voltage. . . . . . . . . . . . . . . . 42

5.5 Completed prototype on solderable breadboard. . . . . . . . . . . . . . 43 
5.6 Measured output voltages (peak-to-peak values). . . . . . . . . . . . . . . . . 44

5.7 Output frequency spectrum for input drive of $90 \mathrm{mV}_{\mathrm{pp}} . \ldots \ldots \ldots \ldots$

5.8 Output frequency spectrum for input drive of $190 \mathrm{mV}_{\mathrm{pp}} . \ldots \ldots \ldots$

5.9 Output frequency spectrum for input drive of $210 \mathrm{mV}_{\mathrm{pp}} . \ldots \ldots \ldots$

5.10 Measured Class AB and Class C collector voltages (peak-to-peak values). . . . . . 46

5.11 Plot of measured power efficiencies. . . . . . . . . . . . . . . . 47

$6.1 \quad$ Schottky-clamped NPN transistor [5]. . . . . . . . . . . . . . . . . . . . . 50

A.1 Bias circuit. Nodes $V \_$base $\_A B$ and Bias_C are the connected to the bases of the Class AB and Class C stages, respectively. . . . . . . . . . . . . . . . . . 51

A.2 Class AB and Class C stages with impedance inverter, matching network, and partial positive feedback implemented using mutually coupled inductors (to simulate the transformer). Nodes $V_{-}$base $\_$AB and Bias $_{-} C$ are the connections to the bias circuit, shown in Figure $\mid$ A.1. $\ldots \ldots \ldots$

B.1 View of PCB top layer. All collector traces are routed on this layer, and a power supply plane covers the unused area . . . . . . . . . . . . . . . . . . . . 53

B.2 View of PCB bottom layer. All base traces are routed on this layer, and a ground plane covers the unused area. . . . . . . . . . . . . . . . . . . . 54 


\section{Chapter 1}

\section{Introduction}

\subsection{Motivation}

New complex, multi-carrier modulation schemes have emerged in the past decade to accommodate faster data rates and an ever-increasing number of wireless device users into the limited bandwidth of the commercial frequency spectrum. These modulation schemes, while effective in maximizing the use of the bandwidth available to users, have presented some challenging problems in the design of RF power amplifiers (PAs).

\subsubsection{Orthogonal Frequency Division Multiplexing (OFDM)}

OFDM is a modulation scheme used in $4 \mathrm{G}$ long-term evolution (LTE) wireless systems that, due to signal characteristics that will now be briefly discussed, is quite difficult to amplify efficiently. OFDM employs a wideband system in which baseband data is modulated (split) into several orthogonal subcarrier channels, which are then summed together for transmission. Due to the independent phases of the subcarriers that often lead to constructive interference, the OFDM signal has what is 
known as a high peak-to-average power ratio (PAPR) ${ }^{1}$, given by

$$
C=\frac{|P|_{\text {peak }}}{P_{\text {rms }}}
$$

where $|P|_{\text {peak }}$ is the peak amplitude of the signal, and $P_{\text {rms }}$ is the RMS (average) value of the signal. To illustrate this phenomenon, a very rudimentary example showing the result of summing sinusoids of varying amplitude and phase to form a high-PAPR signal is given in Figure 1.1. An actual example of an OFDM signal found in an LTE system is shown in Figure 1.2.

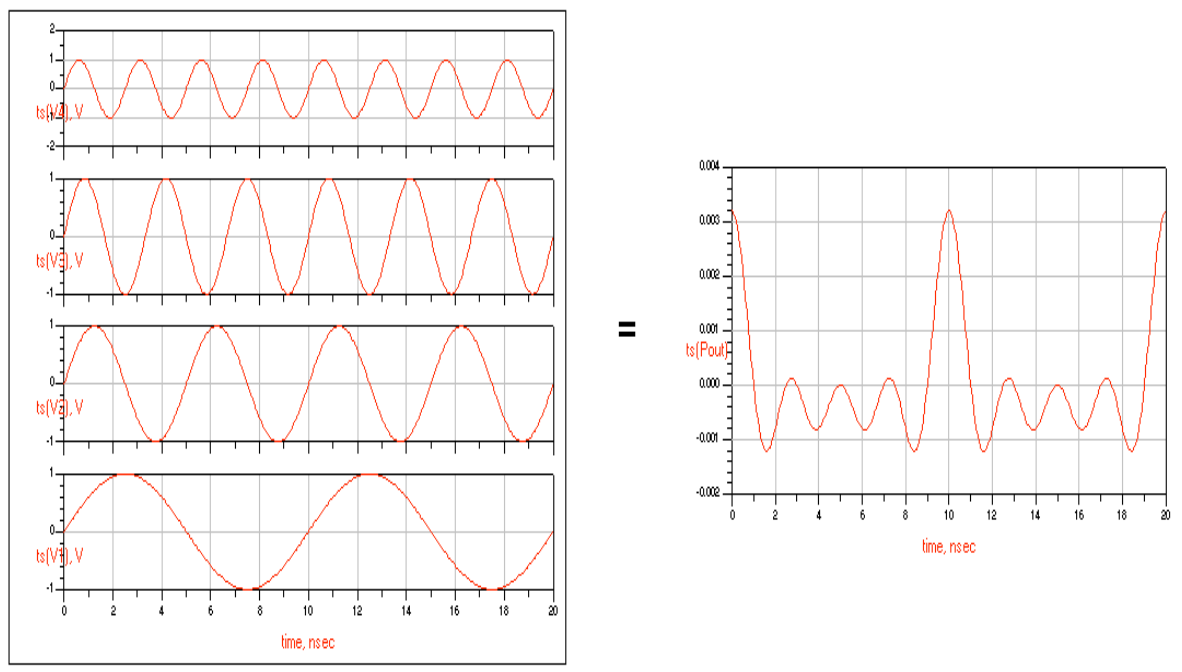

Figure 1.1: Summing of signals with varied amplitude and phase to form a highPAPR signal. [7]

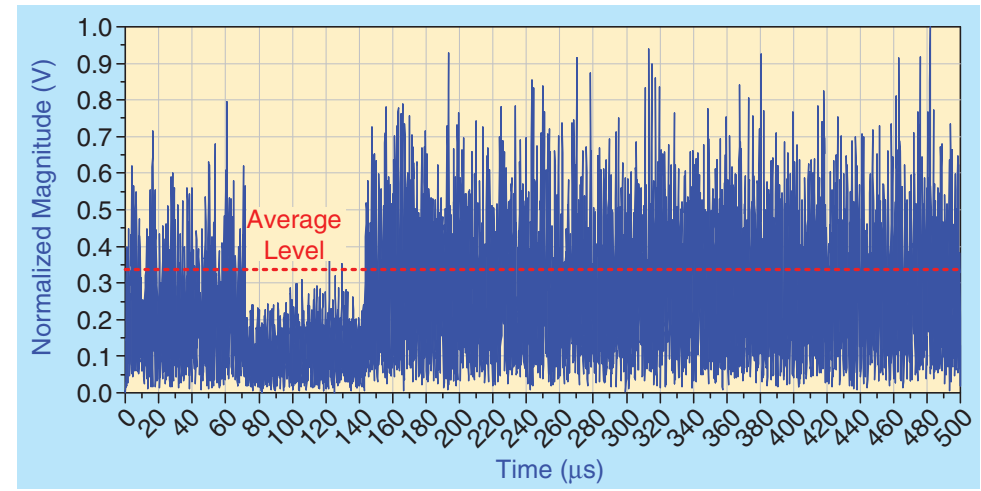

Figure 1.2: Time-domain waveform of an LTE signal. [6]

\footnotetext{
${ }^{1}$ Also known as crest factor $(\mathrm{C})$.
} 


\subsubsection{The Linearity-Efficiency Tradeoff in PA Design}

OFDM signals (as well as those generated in other standards such as WCDMA and WiMAX), while spectrally efficient, are difficult to amplify efficiently using conventional PAs due to their high PAPR. In order to avoid saturation of the amplifier (which will cause spurious out-of-band emissions) and/or exceeding FCC maximum output power restrictions during peak excursions of the modulated input signal, the gain of the amplifier must be "backed-off" from its maximum. There are two metrics for calculating the efficiency of a PA:

$$
\begin{aligned}
\mathrm{PE} & =\frac{P_{\text {out }}}{P_{\mathrm{DC}}} \\
\mathrm{PAE} & =\frac{P_{\text {out }}-P_{\text {in }}}{P_{\mathrm{DC}}}
\end{aligned}
$$

Power efficiency (PE), given by $(1.2)$, is a measure of the percentage of the DC power drawn from the power supply that is converted into useful RF output power. Power-added efficiency (PAE), given by (1.3), uses a ratio of the power gain of the amplifier to the DC power drawn from the supply. A typical PAE curve for a conventional Class AB amplifier is shown in Figure 1.3 .

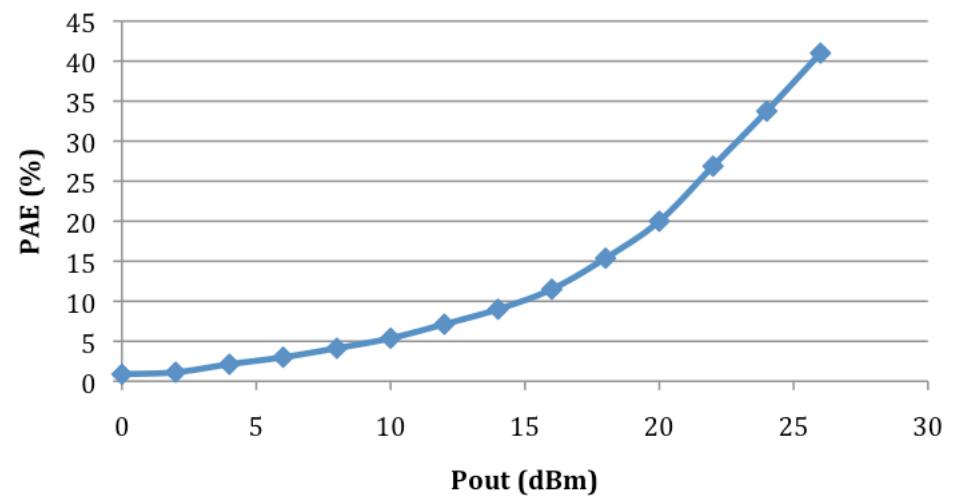

Figure 1.3: Typical PAE curve for conventional Class AB amplifier. [7]

From Figure 1.3 , observe that as one begins to back off on the output power of the amplifier (presumably to avoid linearity degradation), the efficiency begins to drop off substantially. Therefore, the need for a device that is both linear and power-efficient, unlike the conventional Class $\mathrm{AB}$ PA, is quite clear. 


\subsection{Power Amplifier Classes of Operation}

An brief overview of the linear, or current-mode, classes of operation for power amplifiers is required, since these are the focus of this work. Switching-mode power amplifier classes, such as Class E and Class F, are covered extensively elsewhere—see [1] and [9] — but are omitted from the discussion here.

Figure 1.4 shows the conduction angles for the linear classes of operation using a ideal (piecewise linear) transistor I-V characteristic. In Class A, the bias point is such that the transistor conducts current for the full cycle of the input signal, hence giving it a conduction angle of $2 \pi$. The bias point for Class B is at the "knee" of the transistor I-V characteristic, such that the transistor is off for negative excursions of the input signal. This results in a conduction angle of $\pi$ for Class $\mathrm{B}$. Any conduction angles less than $\pi$ are defined as Class C. Class AB (not shown in Figure 1.4) is defined for conduction angles between $\pi$ and $2 \pi$, though the Class AB bias point is often placed right near the knee of the I-V characteristic ${ }^{2}$ A summary of the conduction angles and theoretical maximum efficiencies of each of these classes is given in Table 1.1 .

Table 1.1: Conduction angles and maximum theoretical efficiencies for Class A, $\mathrm{B}$, and $\mathrm{C}$ amplifiers

\begin{tabular}{|c|c|c|}
\hline PA Class & Conduction Angle & Max. Theoretical Efficiency \\
\hline Class $A$ & $\theta=2 \pi$ & $50 \%$ \\
\hline Class $A B$ & $\pi<\theta<2 \pi$ & $78.5 \%$ \\
\hline Class $B$ & $\theta=\pi$ & $78.5 \%$ \\
\hline Class $C$ & $\theta<\pi$ & $100 \%$ \\
\hline
\end{tabular}

\footnotetext{
${ }^{2}$ These classes of operation were defined in the days of vacuum tubes, which display somewhat ideal characteristics. An ideal Class B bias point is rather difficult to achieve using modern solid-state devices (which do not display ideal I-V characteristics), and most designs that target this mode of operation technically operate in Class AB.
} 


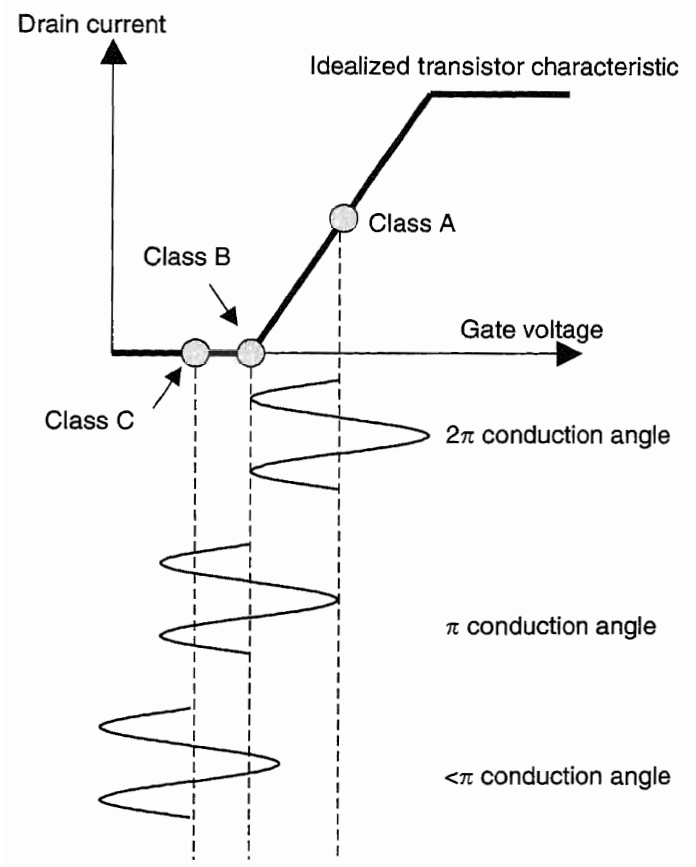

Figure 1.4: Definition of conduction angle for Class A, B, and C operation. [9]
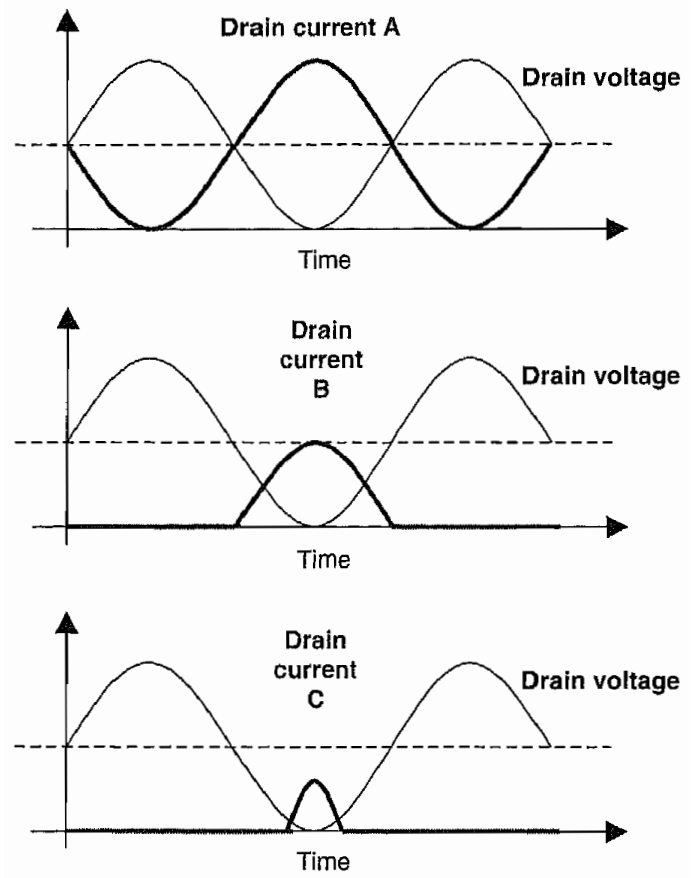

Figure 1.5: Voltage and current waveforms for Class A, B, and C operation. [9] 


\subsection{The Doherty Amplifier}

The Doherty amplifier was conceived in the 1930s by William H. Doherty as a technique for improving the efficiency of high-power tube amplifiers used in AM broadcasting, where hundreds of kilowatts were being dissipated in the tubes (AM signals suffer from significant PAPR). The advent of FM broadcasting, which outputs an essentially constant amplitude signal, caused this circuit to be forgotten for many years, until it was recently resurrected as a method to combat the efficiency degradation caused by today's high-PAPR modulated signals.

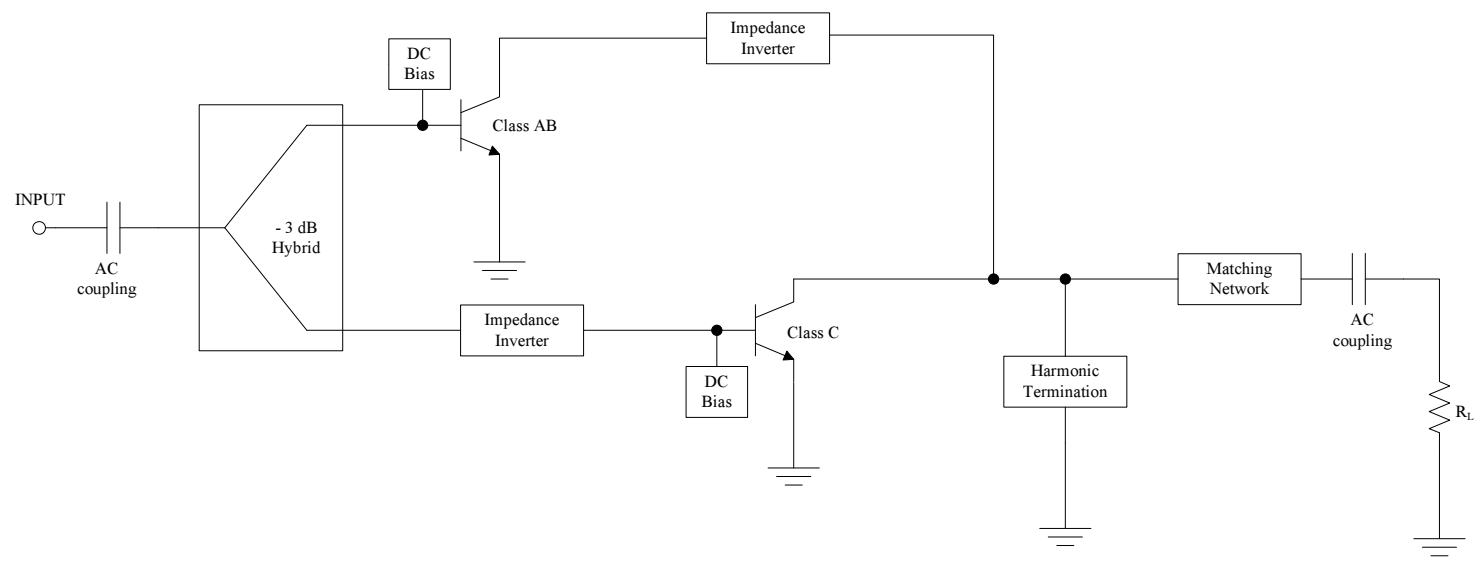

Figure 1.6: Block diagram of Doherty amplifier.

A block diagram of the Doherty amplifier is shown in Figure 1.6. The Class AB amplifier is the "carrier-level" amplifier, and the Class C amplifier is the "peaking" amplifier. When the input signal is at a low power level, the peaking amplifier is off, and only the carrier amplifier is operating. Once the input signal crosses a certain threshold, the peaking amplifier activates and begins to supply current (both amplifiers are active in this case). Due to the action of the impedance inverters [this will be detailed in Section 2.2], the injection of signal by the peaking amplifier will cause the load impedance presented to the carrier amplifier to be decreased; this is often referred to as "active load-pulling" [1] or "load modulation." As the input signal increases, the magnitude of the current injected by the Class $\mathrm{C}$ also increases, thereby reducing the load impedance seen by the carrier stage and allowing it to deliver higher current while maintaing a constant voltage magnitude. 
To make a clear comparison between the topology presented in this paper to the Doherty amplifier, we will refer to this load modulation as the Doherty effect. The detailed theory governing the operation of the Doherty amplifier is covered in [2] and [1], and the reader is encouraged to view these references for detailed information. However, the Doherty effect will be explained in detail, in the context of our topology, in Sections 2.3 and 3.1 .

\subsection{Prior Work}

The general concept behind the negative conductance amplifier that will be presented in this work has been set forth in an existing U.S. patent [8]; however, this patent does not offer a practical implementation of the concept. A previous attempt to implement this topology was unsuccessful, most likely due to the use of cascaded phase-shifting and voltage scaling networks which were highly sensitive to component variation and non-idealities, as well as the absence of harmonic termination [7]. 


\section{Chapter 2}

\section{The Negative Conductance Topology}

\subsection{General Overview}

A block diagram for the proposed amplifier configuration is shown in Figure 2.1.

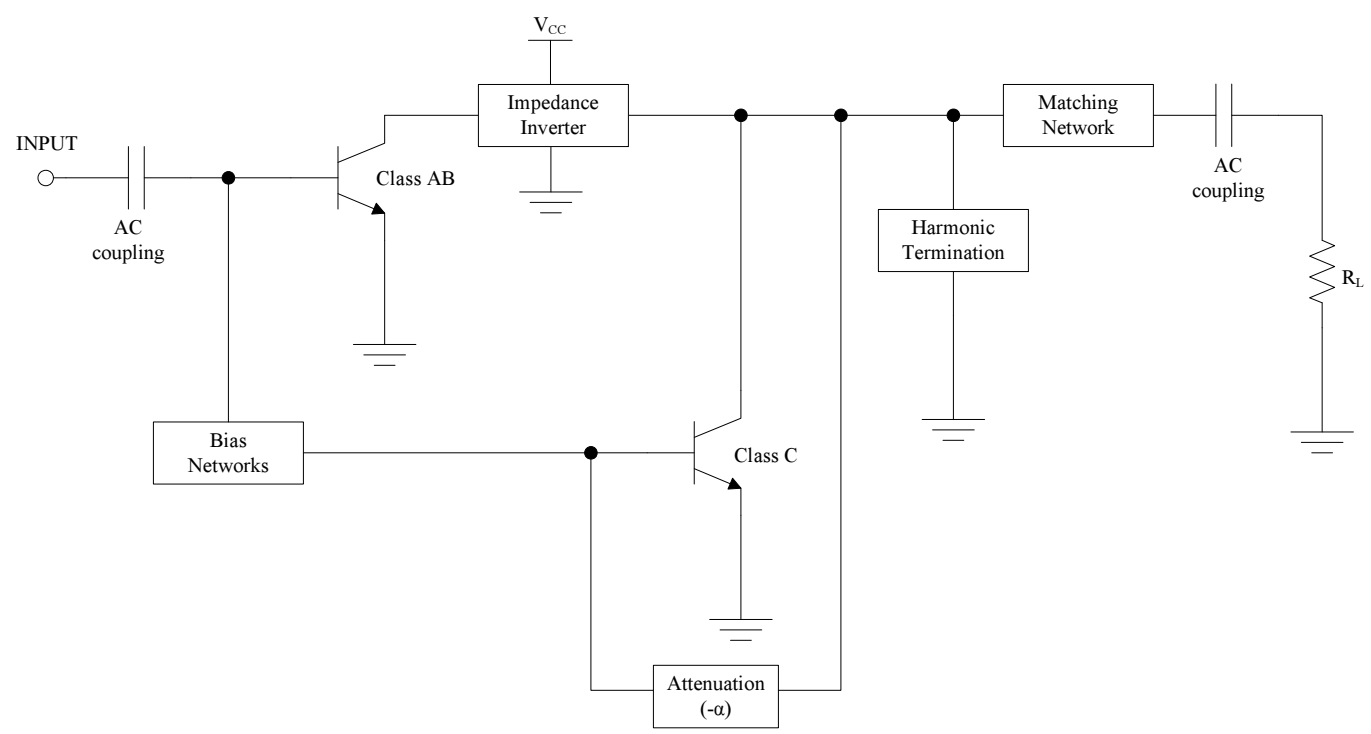

Figure 2.1: Conceptual diagram of amplifier in negative conductance configuration.

The amplifier's input signal is fed into a Class $\mathrm{AB}$ amplifier, which in turn drives the input of an impedance inverter. For purposes that will become clear in Section 2.3 , the voltage developed at 
the output of the impedance inverter is attenuated and inverted (denoted by $-\alpha$ in the figure), and fed back to the input of a Class $\mathrm{C}$ amplifier, which also has its collector terminal connected to the impedance inverter. The output of the impedance inverter is presented with a harmonic trap and a matching network that will provide an impedance match to the load. The following sections will cover each stage of this configuration in detail.

\subsection{The Impedance Inverter}

The impedance inverter is arguably the most important element in the negative conductance configuration, due to certain properties that will be discussed here, and in Section 2.3. An impedance inverter using lumped elements is shown in Figure 2.2, with a load resistance $R$ connected to the output.

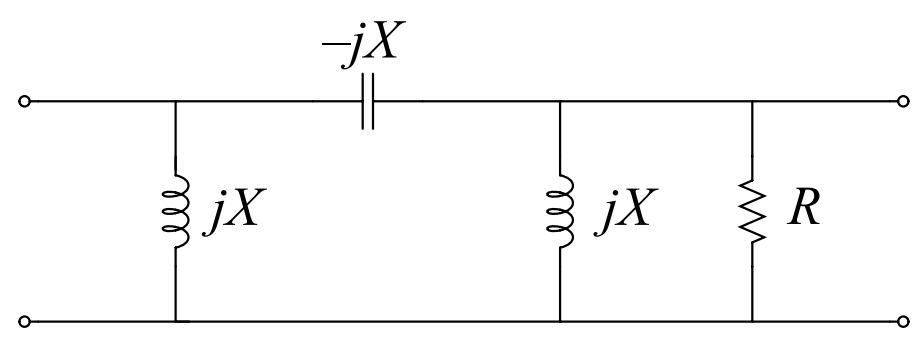

Figure 2.2: Lumped element (LC) impedance inverter.

It is worthy of note that the resistance $R$ in Figure 2.2 is not a physical resistor, but rather the transformed load presented to the impedance inverter output (and hence, the collector of the Class $\mathrm{C}$ device) by the matching network. The impedance inverter is abstracted as a two-port network in Figure 2.3 to clearly illustrate its connection to the Class $\mathrm{AB}$ and Class $\mathrm{C}$ amplifiers. 


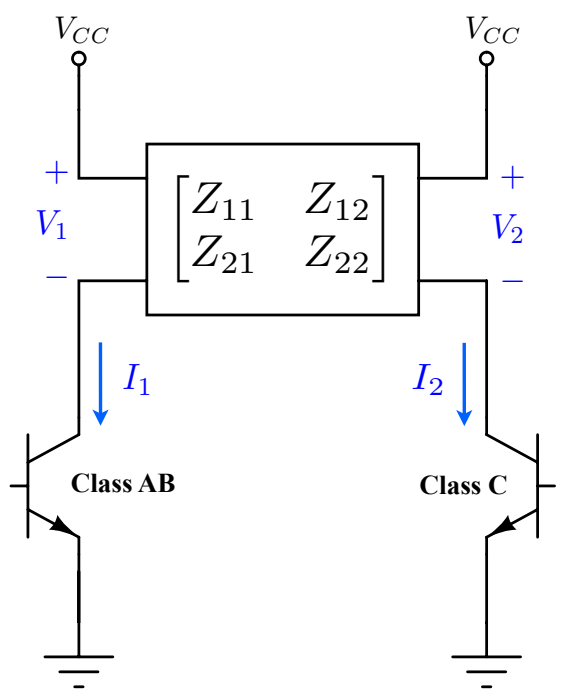

Figure 2.3: Impedance inverter, represented as generic two-port network, connected to the Class AB amplifier (Port 1) and Class $\mathrm{C}$ amplifier (Port 2).

The Z-matrix for the impedance inverter shown above is given in (2.1).

$$
[Z]=\left[\begin{array}{cc}
\frac{X^{2}}{R} & j X \\
j X & 0
\end{array}\right]
$$

Using the Z-matrix to relate the port voltages and currents and substituting the values in (2.1) leads to the important equations (2.2) and (2.3).

$$
\begin{array}{r}
{\left[\begin{array}{l}
V_{1} \\
V_{2}
\end{array}\right]=\left[\begin{array}{ll}
Z_{11} & Z_{12} \\
Z_{21} & Z_{22}
\end{array}\right]\left[\begin{array}{l}
I_{1} \\
I_{2}
\end{array}\right]} \\
V_{1}=Z_{11} I_{1}+Z_{12} I_{2} \\
V_{2}=Z_{21} I_{1}+Z_{22} I_{2} \\
V_{1}=\frac{X^{2}}{R} I_{1}+j X I_{2} \\
V_{2}=j X I_{1}
\end{array}
$$


From (2.3), it is clear that the voltage developed at the collector of the Class $\mathrm{C}$ device, $V_{2}$, is equal to the value of the Class $\mathrm{AB}$ collector current, scaled by the reactance $X$ of the impedance inverter components, and phase shifted by $90^{\circ}$. Note that $V_{2}$, which is presented to the output load through the matching network, is not affected by current perturbations caused by the Class $\mathrm{C}$ device.

Another important characteristic of the impedance inverter is illustrated by $(2.3)$ : the impedance inverter allows the linearity of the overall amplifier to be determined solely by the Class $\mathrm{AB}$ stage. The Class $\mathrm{AB}$ amplifier will have a very high output impedance (ideally, infinite), which the impedance inverter will convert to zero impedance at its output. Therefore, any injection of current at the fundamental into the output of the impedance inverter (i.e. from the Class $\mathrm{C}$ stage) will not affect the voltage and power gains.

\subsection{Achieving the "Doherty Effect" via Transformer Feedback}

Let us assume that the Class $\mathrm{C}$ amplifier shown in Figure 2.3 is driven $90^{\circ}$ out of phase with respect to the Class $\mathrm{AB}$ amplifier; that is, $I_{1}=\left|I_{1}\right| \angle 0^{\circ}$ and $I_{2}=\left|I_{2}\right| \angle 90^{\circ}=j I_{2}$. Under these conditions, and by using the action of the impedance inverter modeled in (2.2), we derive the following result:

$$
\begin{aligned}
V_{1} & =Z_{11} I_{1}+Z_{12} I_{2} \\
& =\frac{X^{2}}{R} I_{1}+j X\left(j I_{2}\right) \\
V_{1} & =\frac{X^{2}}{R} I_{1}-X I_{2}
\end{aligned}
$$

From the second term in 2.4, we observe a mechanism that is key to the operation of this circuit: as $I_{2}$ increases (indicating that the Class $\mathrm{C}$ device has been activated and is pulling collector current), the voltage swing $V_{1}$ of the Class $\mathrm{AB}$ amplifier is reduced. It will now be shown that exploiting this behavior in a controlled manner leads to an elegant solution for preventing saturation of the Class AB amplifier. 
To reiterate, the relation in 2.4 is only valid if the Class $\mathrm{C}$ collector current is $90^{\circ}$ phaseshifted with respect to the Class $\mathrm{AB}$ collector current. Recall from 2.3) that the impedance inverter conveniently provides a $90^{\circ}$ phase shift from input to output. Therefore, if the input voltage for the Class $\mathrm{C}$ device is derived from the output of the impedance inverter, which is driven by the Class $\mathrm{AB}$ amplifier, the following will be true:

- The phase of the input voltage to the base of the Class $\mathrm{C}$ device will be $90^{\circ}$ out of phase with the Class AB collector voltage.

- The magnitude of input voltage to the base of the Class $\mathrm{C}$ device will increase proportionally to the magnitude of the Class $\mathrm{AB}$ collector voltage.

Connecting the impedance inverter output to the base terminal of the Class $\mathrm{C}$ device as outlined above implements the feedback loop shown in the block diagram of Figure 2.1. The only remaining tasks are to attenuate the voltage being fed back onto the Class $\mathrm{C}$ base terminal (the voltage developed at the impedance inverter will be of substantial magnitude and too large to feed directly onto the base of the transistor), and to invert the feedback signal with respect to the impedance inverter output. Attenuation and inversion of the signal are easily accomplished in the method that will now be shown.

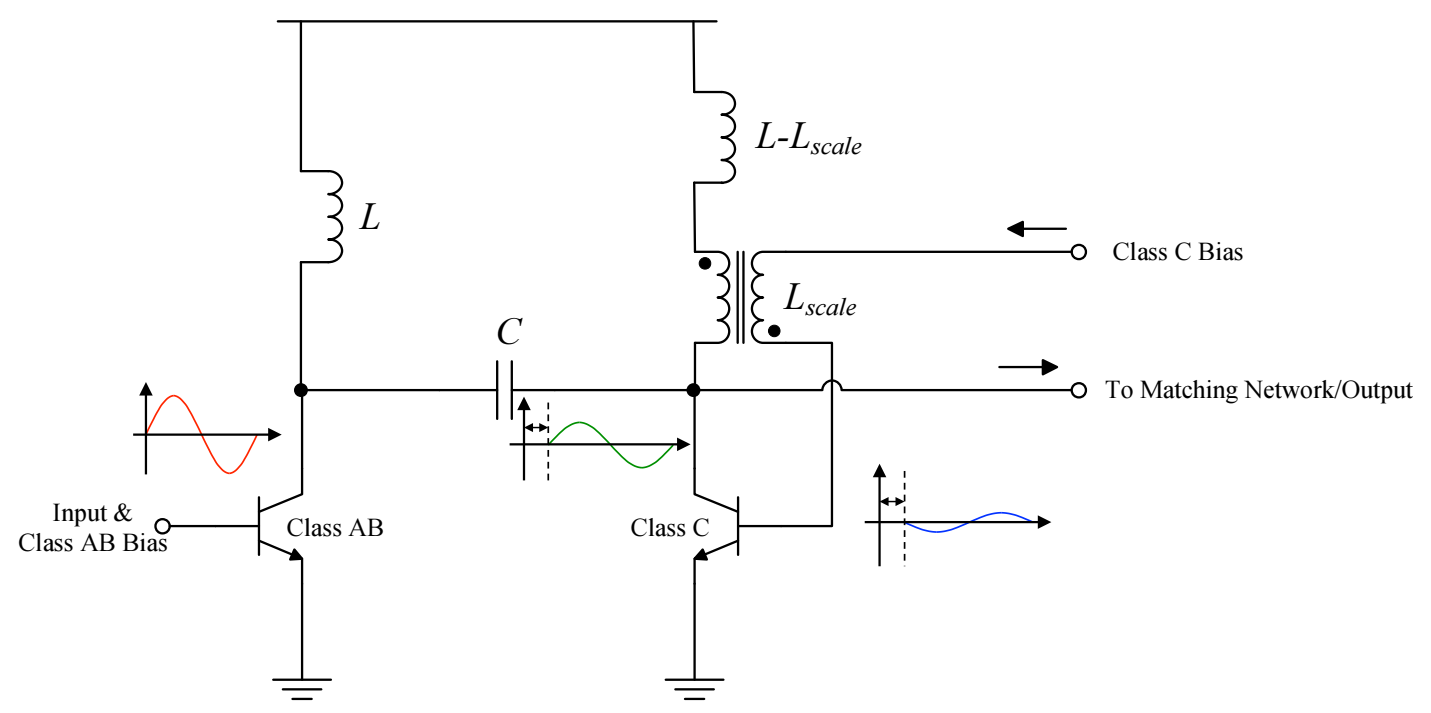

Figure 2.4: Class $C$ partial positive feedback configuration. An inverting transformer is connected in series with the output inductor of the impedance inverter. 
Figure 2.4 shows a feedback configuration, dubbed "partial positive feedback" (PPF) that will implement the above criteria. The output inductor of the impedance inverter is placed in series with

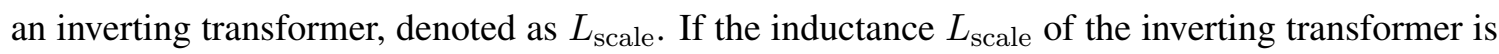
chosen to be very small relative to the larger series inductor $L$, by voltage division, the voltage across it will be a proportionally attenuated 'version' of the impedance inverter output swing. Naturally, since the transformer is inverting, the signal on the secondary side of the transformer (which is connected to the Class $\mathrm{C}$ input), is inverted with respect to the collector. The appropriate sizing of $L_{\text {scale }}$ and $L$ is somewhat dependent on design specifications, and will be covered in more detail in Section 4.5.

It should be noted that the use of a transformer to accomplish PPF is a robust approach, since it is a single, small component (due to the small inductance) that accomplishes both attenuation and inversion of the feedback signal. This is desirable over passive voltage attenuation and phase-shift networks, since these would increase component count in the design, and their proper functionality is heavily dependent on accurate component values. Since the inductance of the transformer in the PPF configuration is very small relative to the larger inductor in series with it, it is relatively insensitive to component variation and non-idealities. 


\section{Chapter 3}

\section{Design Methodology}

The following material aims to present a set of a priori design methods for realizing a negative conductance power amplifier. The transistors used in the design are assumed here to be bipolar; however, the methods should generally apply to any device technology. The presented design methodology also employs techniques that make the design scalable for integrated circuit applications, such as the forming of larger power transistor devices from individual "fingers"-smaller transistors connected in parallel.

\subsection{Active Device Sizing}

The design of a negative conductance amplifier begins with a standard Class AB power amplifier. The sizing $\unlhd^{1}$ and biasing for this stage are chosen for a given set of design specifications-supply voltage, output power, desired transconductance, etc. This is a generic design procedure and is covered in detail in the literature [1]; therefore, it will not be discussed here. As was mentioned in Section 2.2, the linearity and output power of the negative conductance amplifier are determined by the Class AB stage alone, so any specifications applied here will conveniently apply to the entire amplifier.

\footnotetext{
${ }^{1}$ When the term sizing is used henceforth, it will refer to the number of fingers that make up a given power transistor (this will determine the transconductance and current capability of the overall transistor).
} 
Once a Class AB stage suitable for design requirements has been completed, the next step is to replicate the device (i.e., create a new device with equal sizing) and set its bias such that it operates in a Class $\mathrm{C}$ mode. In order to better understand how to determine the proper bias and sizing for the Class $\mathrm{C}$ stage, we must revisit the current and voltage relations between the Class $\mathrm{AB}$ and Class $\mathrm{C}$ stages connected to the impedance inverter and consider an example.

It was previously emphasized that one of the main goals of this design is to prevent saturation of the Class $\mathrm{AB}$ amplifier while maintaining maximum possible efficiency; this implies that it is desirable to hold the Class $\mathrm{AB}$ output voltage constant at its maximum value (before saturation), even as the input voltage continues to increase. The necessary conditions to achieve this behavior are derived below, using the notation from Section 2.2 for the Class $\mathrm{AB}$ voltage and current $\left(V_{1}, I_{1}\right)$ and Class $\mathrm{C}$ voltage and current $\left(V_{2}, I_{2}\right)$. Modifying (2.4) as follows:

$$
\Delta V_{1(\max )}=\frac{X^{2}}{R} \Delta I_{1}-X \Delta I_{2}
$$

where $\Delta V_{1(\max )}$ represents deviation from the maximum Class $\mathrm{AB}$ voltage swing, and setting $\Delta V_{1(\max )}=0$ (constant maximum voltage) yields

$$
\begin{aligned}
\Delta V_{1(\max )}=0 & =\frac{X^{2}}{R} \Delta I_{1}-X \Delta I_{2} \\
& =X\left(\frac{X}{R} \Delta I_{1}-\Delta I_{2}\right) \\
\Delta I_{2} & =\frac{X}{R} \Delta I_{1}
\end{aligned}
$$

The expression in (3.1) contains terms that describe changing collector currents; therefore, it provides a relationship between the transconductances of the Class AB and Class $\mathrm{C}$ stages. Assuming 
equal maximum voltage magnitude at the Class $\mathrm{AB}$ and Class $\mathrm{C}$ nodes yields

$$
\begin{aligned}
V_{1(\max )} & =V_{2(\max )} \\
V_{1(\max )} & =X\left[\frac{X}{R} I_{1(\max )}-I_{2(\max )}\right] \\
V_{2(\max )} & =X I_{1(\max )} \\
X\left[\frac{X}{R} I_{1(\max )}-I_{2(\max )}\right] & =X I_{1(\max )} \\
\frac{X}{R} I_{1(\max )}-I_{2(\max )} & =I_{1(\max )} \\
I_{2(\max )} & =\left[\frac{X}{R}-1\right] I_{1(\max )}
\end{aligned}
$$

Under these conditions, 3.2 shows that the maximum collector current of the two stages are linearly related by a constant involving the ratio between the impedance inverter reactance $X$ and the transformed load resistance $R$ presented by the matching network. This relation is quite convenient, and will be of even further importance when the matching network design is presented in Section 3.4

To illustrate the usefulness of 3.2 , let us now apply it to the case described in the original literature on the Doherty amplifier [2], which calls for the maximum collector currents $I_{1}$ and $I_{2}$ for the two stages to be equal $\left[I_{1(\max )}=I_{2(\max )}\right]$ :

$$
I_{2(\max )}=\left.\left[\frac{X}{R}-1\right] I_{1(\max )}\right|_{I_{1(\max )}=I_{2(\max )}}
$$

The two current terms cancel, reducing the expression to

$$
\begin{gathered}
\frac{X}{R}-1=1 \\
X=2 R
\end{gathered}
$$

Therefore, for equal maximum collector currents, the impedance inverter reactance should be twice 
the value of the transformed load impedance. Also, substituting this result into 3.1] gives

$$
\begin{aligned}
& \Delta I_{2}=\frac{2 R}{R} \Delta I_{1} \\
& \Delta I_{2}=2 \Delta I_{1}
\end{aligned}
$$

With (3.3) and (3.4), there is now enough information to determine the sizing ratio between the Class AB and Class C stages. Specifically, (3.4) indicates that the transconductance of the Class $\mathrm{C}$ stage should be twice that of the Class $\mathrm{AB}$ stage in order to achieve the goal of equal maximum collector currents for both stages. At this point, however, the bias level of the Class $\mathrm{C}$ also needs to be determined.

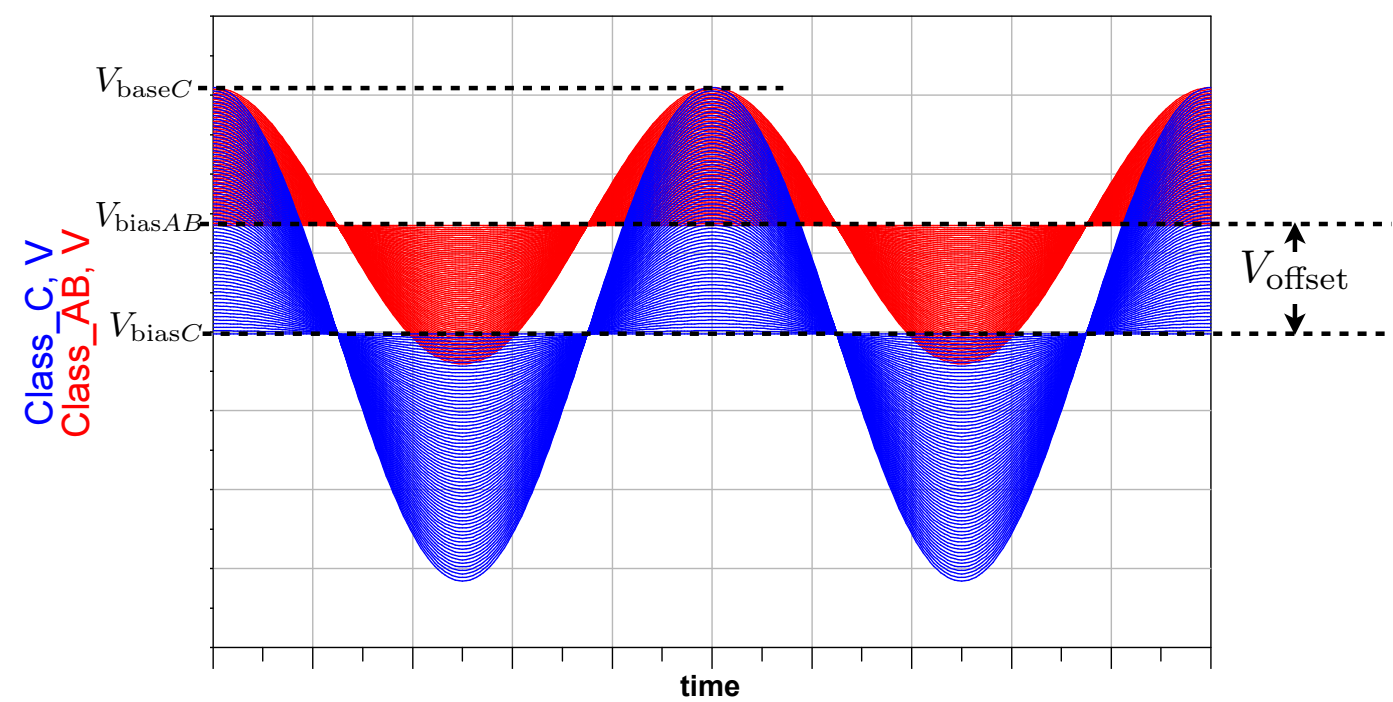

Figure 3.1: Ideal Class $\mathrm{AB}$ and Class $\mathrm{C}$ base voltages of arbitrary frequency, swept over a range of amplitudes. The maximum magnitudes of the Class $\mathrm{AB}$ and Class $\mathrm{C}$ base voltages are equal.

Consider the two ideal sinusoids in Figure 3.1 , where the red waveforms are voltages presented at the base of the Class $\mathrm{AB}$ stage, and the blue waveforms are base voltages applied to the Class C stage. The values $V_{\text {bias } A B}$ and $V_{\text {bias } C}$ represent the $\mathrm{DC}$ bias voltages for the Class $\mathrm{AB}$ stage and Class $\mathrm{C}$ stage, respectively. Recall that the analysis in this section initially began with Class $\mathrm{AB}$ and Class $\mathrm{C}$ stages of equal size, meaning that their transconductances are equal for the time being. 
Naturally, for equally-sized devices to have the same maximum output current, their maximum input voltages should also be equal, regardless of DC bias voltages, as is shown in Figure 3.1. A key design variable, denoted as $V_{\text {offset }}$, is defined as the difference between the Class $\mathrm{AB}$ bias and the Class $\mathrm{C}$ bias:

$$
V_{\text {offset }}=V_{\text {bias } A B}-V_{\text {bias } C}
$$

Using $V_{\text {offset }}$ and the maximum input voltage $\left(V_{\mathrm{in} A B}\right)$ determined previously from the design of the Class $\mathrm{AB}$ stage, a useful relation for ensuring that the magnitude of the Class $\mathrm{C}$ input voltage $\left(V_{\mathrm{base} C}\right)$ is equal to that of the Class $\mathrm{AB}$ is formulated as

$$
V_{\text {base } C}=V_{\text {in } A B}\left[1+\frac{V_{\text {offset }}}{V_{\text {in } A B(\max )}}\right]-V_{\text {offset }}
$$

Notice that 3.6 is a function of the Class $\mathrm{AB}$ input voltage; this effectively models the partial positive feedback mechanism that was presented in Section 2.3. There will likely be multiple combinations of $V_{\text {offset }}$ and Class $C$ device sizing that will meet the design criteria, so it is best to employ an iterative approach to find a suitable combination of these design parameters. Many circuit simulators, such as Agilent ADS, contain equation-based ideal voltage sources and the ability to set design variables for optimization. If such tools are available, the designer can easily perform the following:

- Place equally-sized Class AB and Class C devices in schematic.

- Drive the Class AB input with an ideal voltage source swept over a range of values up to the maximum designed input voltage $\left(V_{\operatorname{in} A B}\right)$

- Create an editable variable for $V_{\text {offset }}$

- Drive the Class $\mathrm{C}$ input with an equation-based ideal voltage source that implements (3.6), using the $V_{\text {offset }}$ variable

- Using Harmonic Balance or other simulation method, plot the fundamental component of the collector current for both stages over the range of input voltages. 
- Simultaneously adjust the size of the Class C stage (adding/removing fingers from the device as necessary) and the value of $V_{\text {offset }}$ until the desired transconductance curves are obtained for the design specifications.

\subsection{Impedance Inverter Sizing}

Since the output linearity of the negative conductance amplifier is dependent upon the Class $A B$ amplifier, the fundamental component of the Class $\mathrm{AB}$ current should be used to calculate the required impedance inverter reactance $X$ to provide the desired output voltage swing. This is a simple calculation using Ohm's law,

$$
X=\frac{V_{1 p(\max )}}{I_{1(\max )}}
$$

where $V_{1 p(\max )}$ is the desired peak magnitude of the Class $\mathrm{AB}$ output voltage, and $I_{1(\max )}$ is the collector current at maximum drive.

\subsection{Bias Circuit}

Standard resistor biasing of RF power amplifiers implemented in bipolar technology is not a suitable practice, due to the high current demands at the base of the amplifying device for higher input drive levels. If suitable protection of the bias generation is not implemented against these increasing DC currents, such as those shown in Figure 3.2 (an actual simulation plot of the DC component of the amplifier base currents for the design given in Chapter 4], the bias circuit may become loaded down and cease to provide the necessary DC voltage to maintain the desired class of operation for the power amplifier. A proposed bias circuit that addresses these "de-biasing" issues is shown in Figure 3.3 


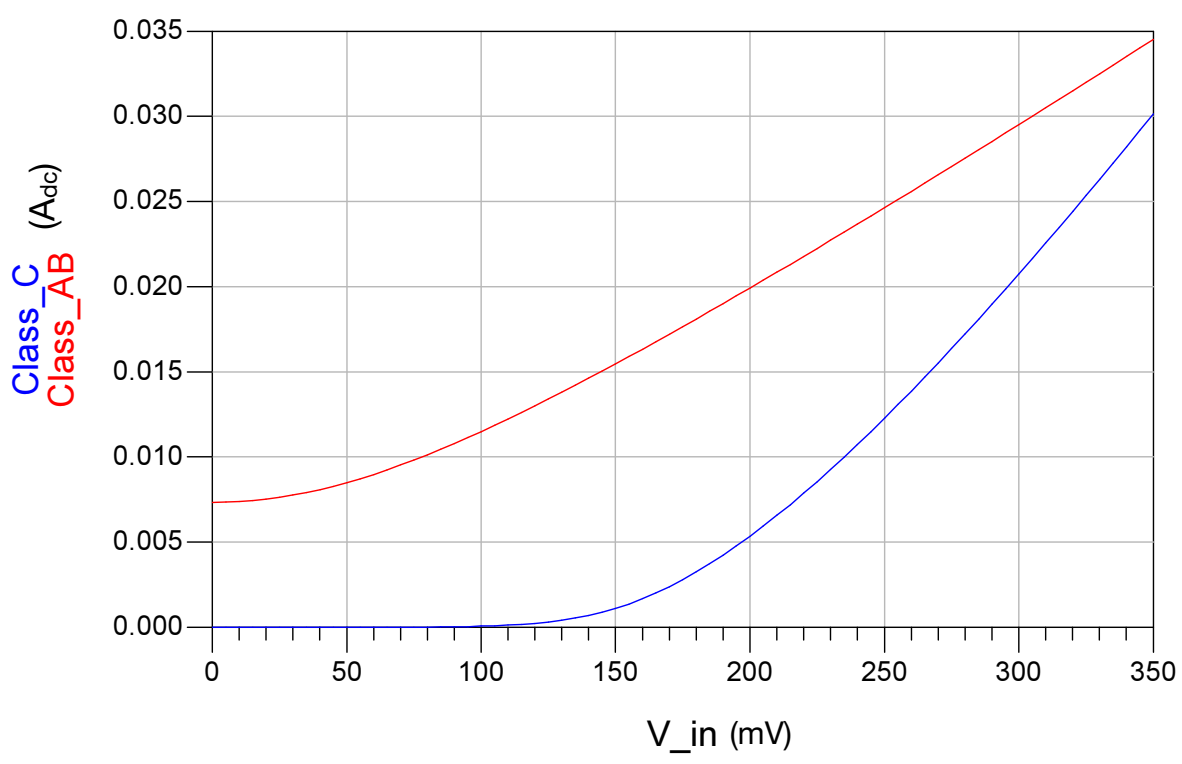

Figure 3.2: Simulation plot of $D C$ component of Class $A B$ and Class $C$ base currents for the design presented in Chapter 4 .

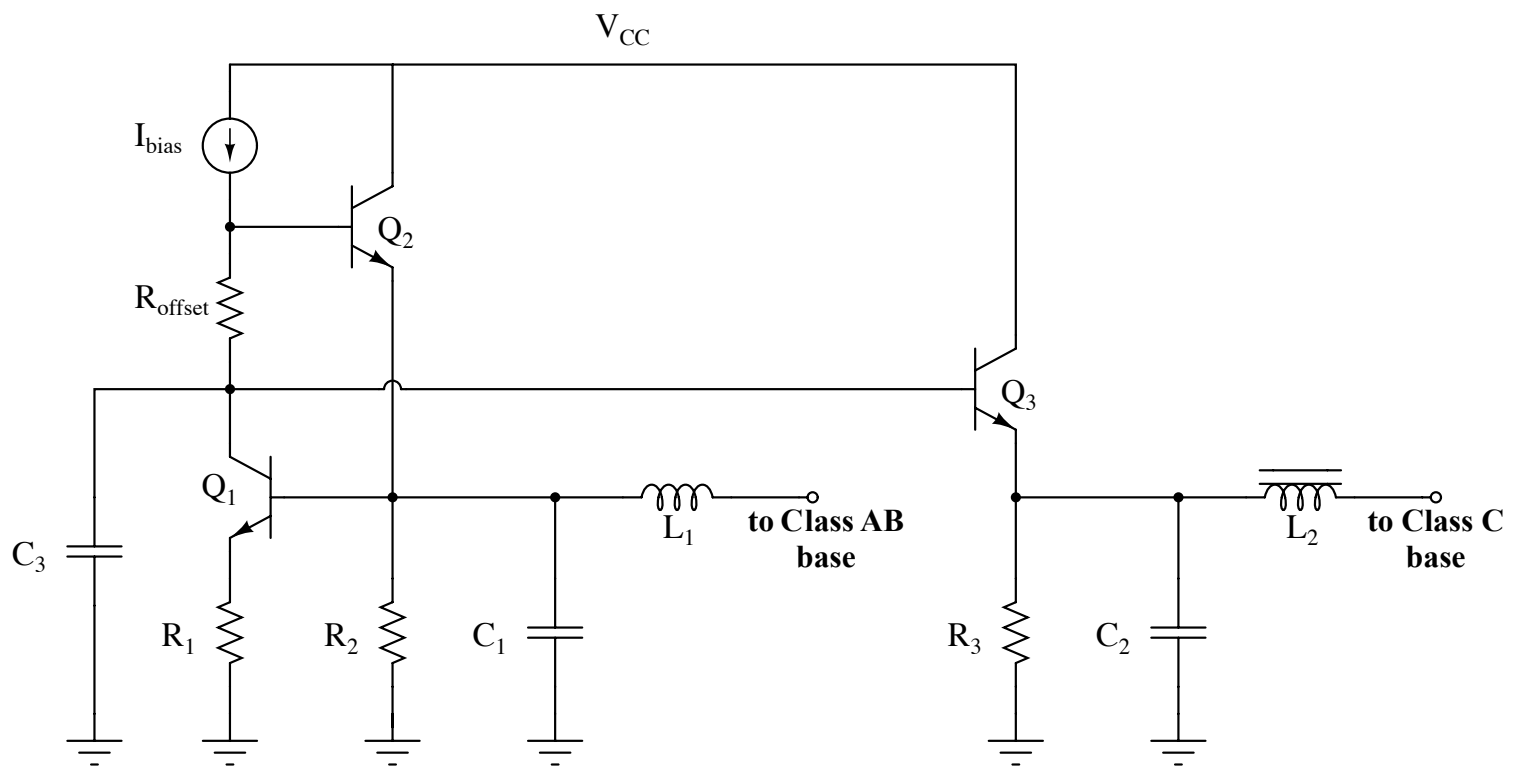

Figure 3.3: Proposed bias circuit.

A list of the components in the bias circuit of Figure 3.3 and their functions is provided in Table 3.1 . 
Table 3.1: List of components in the proposed bias circuit of Figure 3.3 and their respective functions.

\begin{tabular}{|c|c|}
\hline Component & Function \\
\hline Q1 & $\begin{array}{l}\text { Forms a current mirror with both the Class } \mathrm{AB} \text { and Class } \mathrm{C} \text { transistors (not } \\
\text { shown) }\end{array}$ \\
\hline Q2, Q3 & $\begin{array}{l}\text { Emitter followers for buffering of the DC bias outputs. The high input } \\
\text { impedances keep the bias branch of the current mirror isolated from the Class } \\
\text { AB and Class } C \text { transistors, and the output of the emitter followers present a low } \\
\text { impedance path to the transistor base terminals. }\end{array}$ \\
\hline $\mathrm{I}_{\text {bias} 1}$ & $\begin{array}{l}\text { Current source to provide bias current determined previously in the design of the } \\
\text { Class AB stage. }\end{array}$ \\
\hline $\mathrm{R}_{\text {offset }}$ & Implements a voltage drop equal to $V_{\text {offset }}$ for the bias current provided by $\mathrm{I}_{\text {bias1 }}$. \\
\hline $\mathrm{R} 1$ & $\begin{array}{l}\text { Emitter degeneration to match that of the Class } \mathrm{AB} \text { and Class } \mathrm{C} \text { amplifiers. If no } \\
\text { emitter degeneration is used, } \mathrm{R} 1 \text { should be excluded. }\end{array}$ \\
\hline $\mathrm{R} 2, \mathrm{R} 3$ & $\begin{array}{l}\text { Biasing resistors for the emitter followers Q2 and Q3, to ensure that these devices } \\
\text { are activated. }\end{array}$ \\
\hline $\mathrm{C} 1, \mathrm{C} 2$ & $\begin{array}{l}\text { Filtering capacitors, to remove any AC fluctuations or noise from the bias net- } \\
\text { work output }\end{array}$ \\
\hline $\mathrm{C} 3$ & $\begin{array}{l}\text { Compensation capacitor. The combinations of Q1 and Q2, and Q1 and Q3, can } \\
\text { be viewed as a cascade of common-emitter and common-collector stages con- } \\
\text { nected via feedback; the compensation capacitor can be chosen to provide sta- } \\
\text { bility of this feedback loop, and prevent oscillation in the bias circuit. }\end{array}$ \\
\hline L1 & $\begin{array}{l}\text { Large-valued inductor (preferably a ferrite bead or other RF choke) to suppress } \\
\text { high-frequency noise and provide a short-circuit path for DC base current to the } \\
\text { Class AB amplifier. }\end{array}$ \\
\hline $\mathrm{L} 2$ & $\begin{array}{l}\text { Secondary side of of the inverting transformer used for partial positive feedback, } \\
\text { through which the Class C input signal is coupled onto the base (see Section } 2.3 \text {. }\end{array}$ \\
\hline
\end{tabular}


The selection of the component values for the bias circuit is entirely design-dependent, so there may be some trial-and-error involved in selecting the proper values for each component.

\subsection{Matching Network with Integrated Harmonic Trap}

The pi-section low pass matching network (Figure 3.4) is commonly employed in power amplifier designs [1], and provides some elegant intrinsic functionality that will be shown in this section. Note that $C_{\text {couple }}$ provides $\mathrm{AC}$ coupling to the load, and will be chosen to have a large value compared to the other capacitors in the network; therefore, it can be effectively neglected from the analysis.

The design of these matching networks is easily accomplished for given input and load impedances and quality factor $(\mathrm{Q})$ using a Smith chart, and is well-documented in the literature [4]. The matching network for this design will be used to transform the load impedance $R_{L}$ to a suitable impedance $R$ presented to the impedance inverter output at the fundamental frequency, in order to achieve proper negative conductance functionality. Recall from Section 2.3 that the Class AB and Class C current magnitudes are related via a ratio of $X$ to $R$. Therefore, if the desired ratio between the collector currents is known, and the reactance $X$ to provide the necessary Class AB output swing has been calculated using (3.7), the necessary value of the transformed load impedance $R$ can be solved from $(3.2)$.

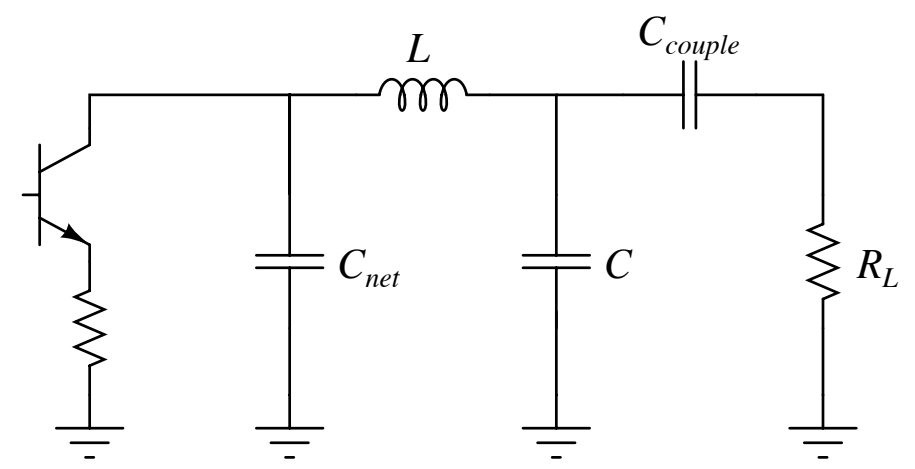

Figure 3.4: Base topology for Pi-section matching network.

After designing a "base" matching network as shown in Figure 3.4 to transform the load $R_{L}$ to 
input impedance $R$ at the fundamental, the network can be modified to include an integrated harmonic termination without altering the network response or matching, as will now be shown.

We know from Section 1.2 that a Class $\mathrm{AB}$ power amplifier output has a significant second harmonic component, which must be properly terminated in order to avoid linearity degradation. Let $X_{\text {net }}$ be the reactance of capacitor $C_{\text {net }}$ in Figure 3.4 at the fundamental. It is well-known that a series LC network will appear as a short circuit at resonance, and will have some net reactance $X_{\text {net }}$ given by

$$
\begin{aligned}
X_{C s}+X_{L s} & =X_{n e t} \\
-\frac{1}{\omega_{0} C_{s}}+\omega_{0} L_{s} & =X_{n e t}
\end{aligned}
$$

where $X_{C s}$ and $X_{L s}$ are the reactances of the series capacitor and inductor, respectively, at the resonant frequency. Therefore, if a series LC network is tuned such that its net reactance at resonance is equal to the net reactance $X_{\text {net }}$ of the shunt capacitor $C_{\text {net }}$ in Figure 3.4, and its resonant frequency is set to the second harmonic as follows,

$$
\frac{1}{\sqrt{L_{s} C_{s}}}=2 \omega_{0}
$$

capacitor $C_{\text {net }}$ can be replaced with a harmonic "trap" that will allow the second harmonic component of the voltage to be shorted to ground as shown in Figure 3.5

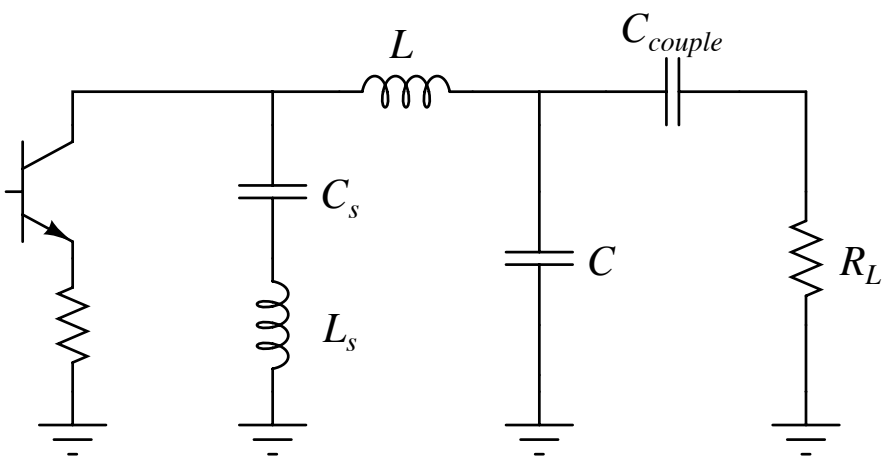

Figure 3.5: Pi-section matching network with integrated harmonic trap 
Solving (3.8) and (3.9) simultaneously yields the design equations for integrating a second harmonic trap into the matching network in Figure 3.5 .

$$
\begin{gathered}
C_{s}=-\frac{3}{4 \omega_{0} X_{n e t}} \\
L_{s}=\frac{1}{4 \omega_{0} C_{s}}
\end{gathered}
$$

\subsection{Class C Transformer Feedback}

The partial positive feedback configuration for the Class $\mathrm{C}$ stage is shown again in Figure 3.6 .

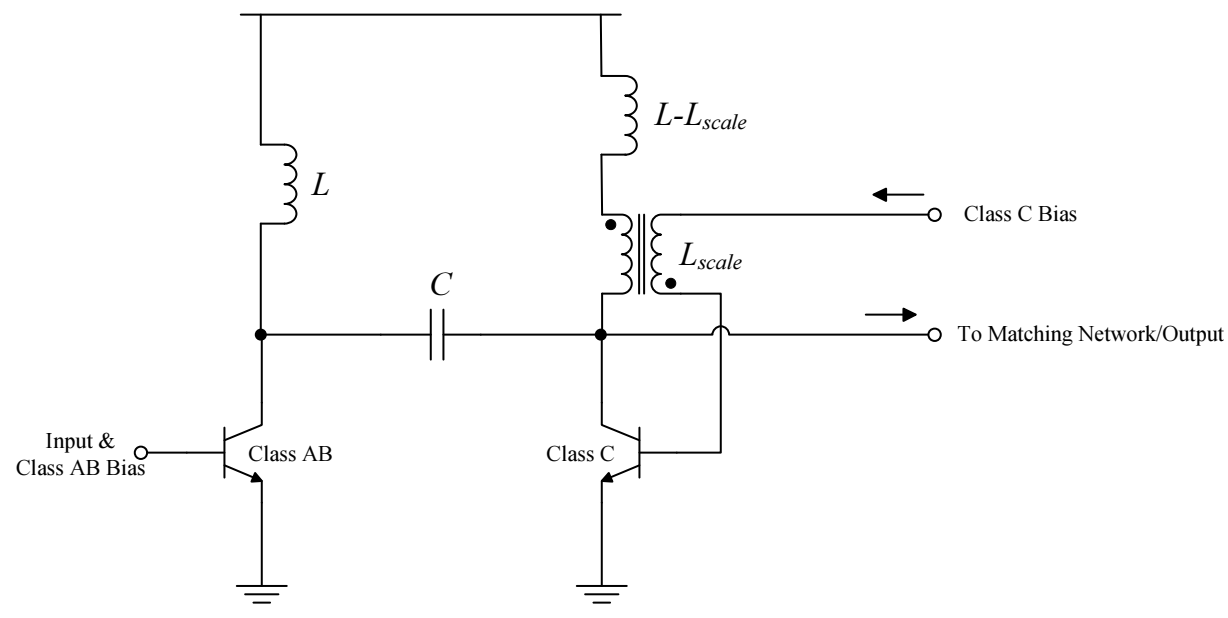

Figure 3.6: Class $\mathrm{C}$ partial positive feedback configuration.

The values of $\mathrm{L}$ and $\mathrm{C}$ in the impedance inverter are easily calculated once the desired $X$ has been determined. The inductance $L_{\text {scale }}$ of the inverting transformer must be calculated such that the voltage developed across it is equal in magnitude to $V_{\text {base } C}$ given by (3.6). Using voltage division of $V_{2}$ at the impedance inverter output results in

$$
V_{\text {base } C}=V_{2} \frac{L_{\text {scale }}}{L+L_{\text {scale }}}
$$

It is then necessary to subtract the calculated value for $L_{\text {scale }}$ from the inductance $L$ in series with it, so that their series combination adds up to a total inductance of $L$; that is, $\left(L-L_{\text {scale }}\right)+L_{\text {scale }}=L$. 
This ensures proper functionality of the impedance inverter, and will not affect the voltage division by any appreciable degree since $L_{\text {scale }}$ is very small with respect to $L$.

\subsection{Summary of Design Procedures}

A step-by-step summary of the design procedures discussed in this chapter is now presented to aid the designer in the design of a negative conductance amplifier using partial positive feedback.

1. Design Class $A B$ amplifier for given set of design specifications (supply voltage, output power, etc.) and verify proper functionality in simulation.

2. Replicate device used in Class $\mathrm{AB}$ amplifier (this will be used as the Class $\mathrm{C}$ stage) and lower its DC bias such that it falls into a Class $\mathrm{C}$ mode (this bias is initially arbitrary).

3. Identify desired ratio between Class $\mathrm{AB}$ and Class $\mathrm{C}$ maximum collector currents and use (3.2) to determine the required value of $X$ in terms of $R$.

4. Substitute the symbolic result for $X$ (in terms of $R$ ) from the previous step into (3.1) to determine the required ratio between the Class $\mathrm{AB}$ and Class $\mathrm{C}$ transconductances.

5. Use idealized computer simulation with equation-based voltage source as outlined in Section 3.1 to iteratively find a combination of $V_{\text {offset }}$ [defined in (3.5)] and Class $\mathrm{C}$ device sizing that shows the desired maximum current and transconductance characteristics identified in Steps 3 and 4 .

6. Implement the bias circuit of Section 3.3 to provide the Class AB bias current determined from the design of Step 1, as well as the value of $V_{\text {offset }}$ calculated in Step 5 between the Class $\mathrm{AB}$ and Class $\mathrm{C}$ bias levels. Choose other components (bias resistors, filtering capacitors, etc.) appropriately for the specific design.

7. Use the magnitude of the maximum fundamental collector current for the Class AB stage and the desired maximum voltage swing to calculate the required value of $X$ using (3.7). 
8. Substitute the value of $X$ from Step 7 into 3.2 to solve for the required value of $R$.

9. Design a "base" pi-section matching network using a Smith chart that will transform match the load $R_{L}$ to the calculated value of $R$ from Step 8 at the fundamental. Use (3.10) and (3.11) to replace shunt capacitor $C_{\text {net }}$ in the base matching network topology with a suitable second harmonic trap.

10. Use (3.6) to determine the required magnitude of the signal being fed back onto the Class $\mathrm{C}$ input $\left(V_{\mathrm{base} C}\right)$ from the impedance inverter output. Then substitute this value into 3.12 to determine the correct transformer inductance $L_{\text {scale }}$ to achieve the required feedback.

11. Optimize the design further to fine tune specific design requirements. 


\section{Chapter 4}

\section{Prototype Design}

A prototype design was created to demonstrate the validity of the design methods presented in Chapter 3 Since the goal of this research was not to realize a working device at normal mobile wireless (low GHz-range) operating frequencies, but rather to demonstrate the validity of the concept as a whole, a low operating frequency of $1 \mathrm{MHz}$ was chosen in order to alleviate any layout challenges and/or device parasitics that may have triggered "false negatives" (i.e., that the circuit topology as a whole is not valid). Similar low-frequency verification of RF power amplifiers has been successful in other research [3].

In order to maintain the IC-like approach of forming power transistors from multiple fingers in parallel, the LM3046 BJT array from National Semiconductor was employed in the prototype design. The LM3046 features 5 NPN BJT devices on a common monolithic substrate. The motivation for choosing these devices was that each of the five transistors on each chip would be closely matched (electrically and thermally) and would provide a reasonable substitute to implementing the design on an integrated circuit. A DC supply of $8 \mathrm{~V}$ was used for the design in order to prevent exceeding any of the maximum rated junction voltages listed in the LM3046 data sheet. 


\subsection{Class AB Stage}

Arbitrarily, the Class $\mathrm{AB}$ stage was chosen to have 10 fingers connected in parallel: five transistors on each of two LM3046 chips. The bias current for the Class AB amplifier was selected as $700 \mu \mathrm{A}$ (resulting in a DC bias voltage of $669 \mathrm{mV}$ ) to give the transconductance curve shown in Figure 4.1

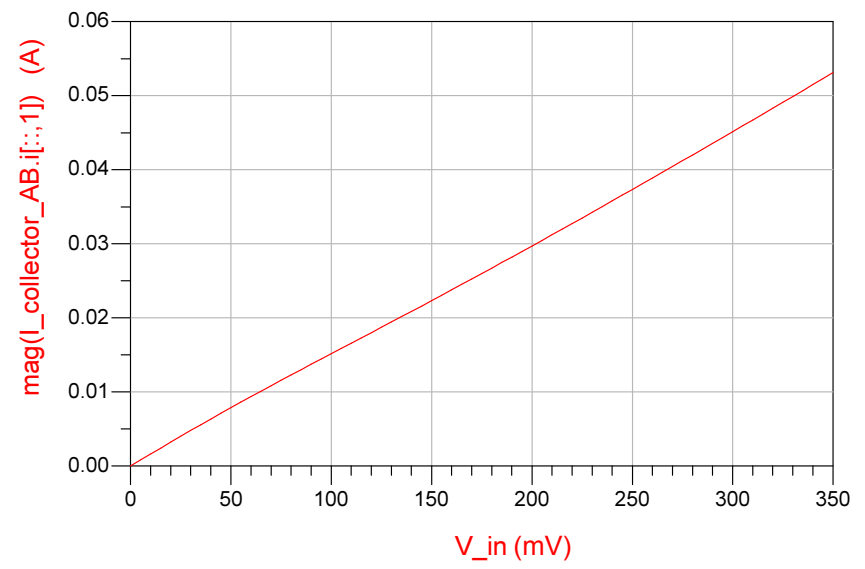

Figure 4.1: Magnitude of Class $\mathrm{AB}$ collector current (fundamental component) vs. input voltage.

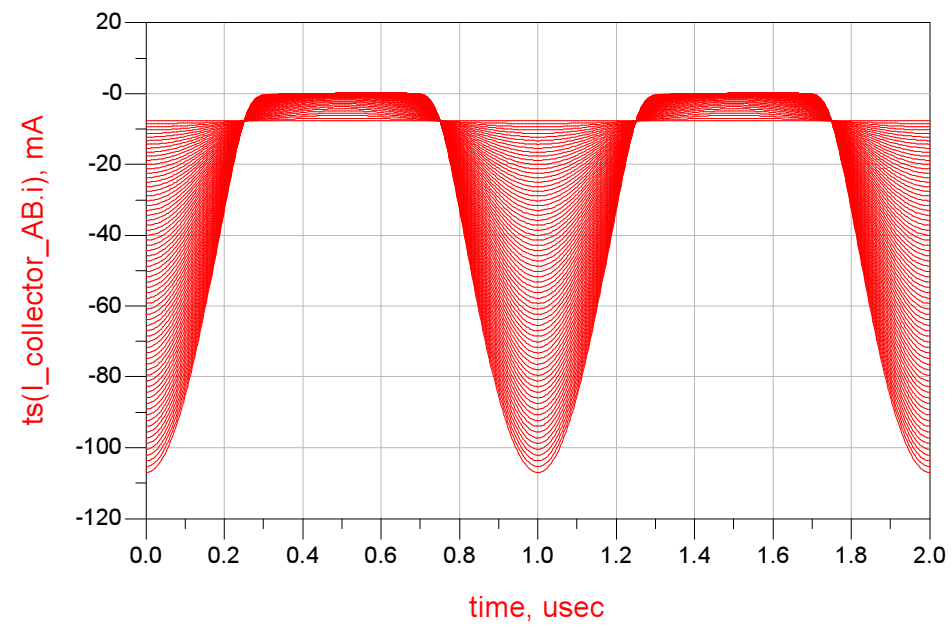

Figure 4.2: Class $\mathrm{AB}$ collector current (time-domain) for input voltages swept from $0 \mathrm{mV}$ to $350 \mathrm{mV}$.

\footnotetext{
${ }^{1}$ Recall that the impedance inverter and matching network are operational only at the fundamental frequency; therefore, only the fundamental component $(1 \mathrm{MHz})$ of the collector current is plotted in Figure 4.1
} 


\subsection{Impedance Inverter}

Choosing the magnitude of the maximum voltage swing for the Class $A B$ to be $\pm 7 \mathrm{~V}$, and using the maximum fundamental current of $53.09 \mathrm{~mA}$ for the Class $\mathrm{AB}$ stage, the required impedance inverter reactance $X$ should be

$$
X=\frac{9 \mathrm{~V}}{53.09 \mathrm{~mA}} \approx 130 \Omega
$$

according to 3.7). At the operating frequency of $1 \mathrm{MHz}$, this results in the following capacitor and inductor values:

$$
\begin{aligned}
L & =\frac{X}{\omega}=20.69 \mu \mathrm{H} \\
C & =-\frac{1}{\omega X}=1.2 \mathrm{nF}
\end{aligned}
$$

To implement the classic Doherty functionality described in Section 3.1[Equations (3.3) and [3.4]], the required value of $R$ seen by the output of the impedance inverter is

$$
R=\frac{X}{2}=65 \Omega
$$

which will be the target for the matching network design.

\subsection{Class C Biasing and Sizing}

A suitable bias point and device sizing were chosen using the equation-based voltage source strategy covered in Section 3.1 and shown in Figure 4.3 The initial sizing of the Class $\mathrm{C}$ was equal to that of that of the Class $\mathrm{AB}$ (10 fingers), and was then increased to 12 fingers total after iteration. A suitable value of $V_{\text {offset }}=275 \mathrm{mV}$ was also found by iteration. The results of this idealized simulation are shown in Figure 4.4 


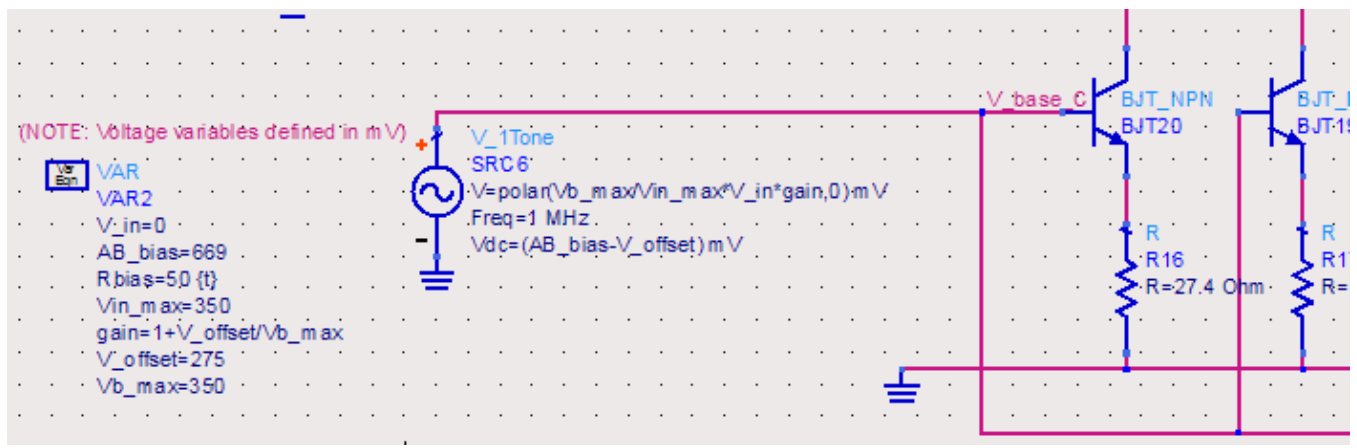

Figure 4.3: Ideal equation-based driving source for Class $\mathrm{C}$ stage in Agilent ADS.
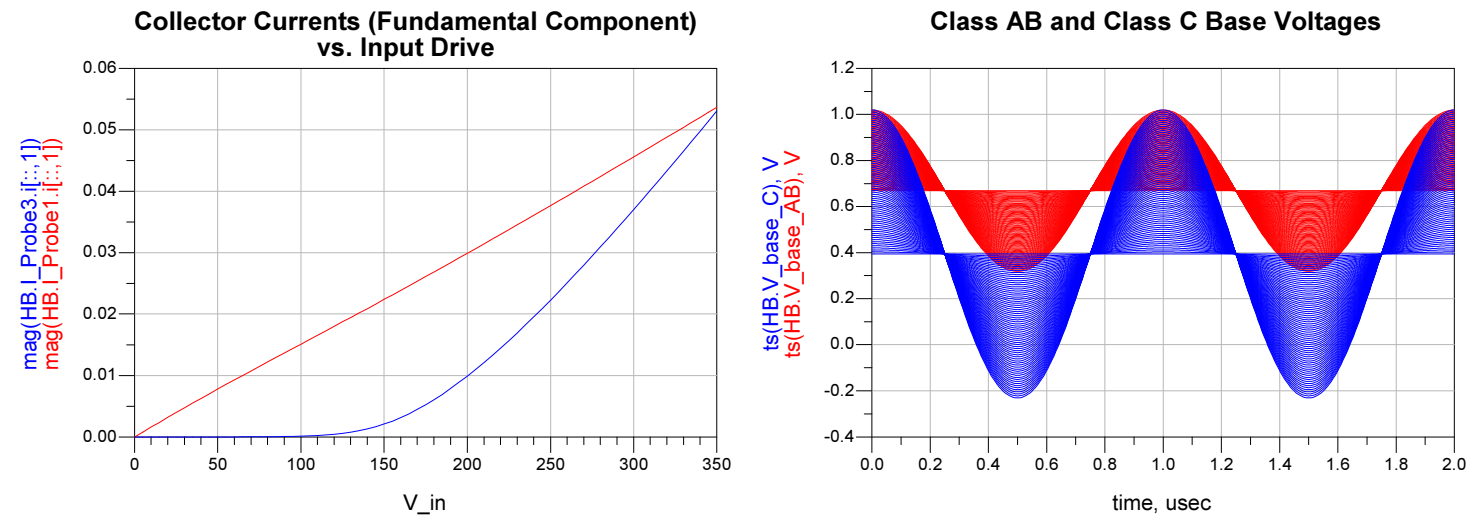

Figure 4.4: Results of idealized simulation to determine correct Class $\mathrm{C}$ biasing and device sizing.

This combination of bias point and device sizing yield the desired 2:1 transconductance ratio between the Class $\mathrm{C}$ and Class $\mathrm{AB}$ stages specified by (3.4).

The bias circuit of Section 3.3 was implemented to output a Class AB bias voltage of $669 \mathrm{mV}$ and a Class $\mathrm{C}$ bias voltage of $394 \mathrm{mV}\left[V_{\text {bias } A B}-V_{\text {offset }}=669 \mathrm{mV}-275 \mathrm{mV}=394 \mathrm{mV}\right]$. The component values chosen for the bias network are given in Table 4.1. Notice in particular that the value of $\mathrm{R}_{\text {offset }}$ is calculated to provide a voltage drop of $275 \mathrm{mV}$ for the bias current of $700 \mu \mathrm{A}$. $100 \mathrm{nF}$ capacitors were verified via simulation to provide sufficient filtering. A ferrite bead was not available when the project was to be built; therefore it was substituted by a large-valued inductor and a small capacitor in parallel (set to be resonant at the fundamental), in order to simulate the behavior of an RF choke or ferrite bead. The chosen value for the transformer $\mathrm{L}_{2}$ will be explained in Section 4.5 , 
Table 4.1: List of component values for the implemented bias circuit (Refer to Figure 3.3 and Table 3.1).

\begin{tabular}{|c|c|}
\hline Component & Value \\
\hline $\mathrm{I}_{\text {bias1 }}$ & $700 \mu \mathrm{A}$ \\
\hline $\mathrm{R}_{\text {offset }}$ & $392 \Omega$ \\
\hline $\mathrm{R}_{1}$ & $27 \Omega$ \\
\hline $\mathrm{R}_{2}$ & $6.81 \mathrm{k} \Omega$ \\
\hline $\mathrm{R}_{3}$ & $2.74 \mathrm{k} \Omega$ \\
\hline $\mathrm{C}_{1}, \mathrm{C}_{2}$ & $100 \mathrm{nF}$ \\
\hline $\mathrm{C}_{3}$ & $4.7 \mu \mathrm{F}$ \\
\hline $\mathrm{L}_{1}$ & $8 \mu \mathrm{H}$ \\
\hline $\mathrm{L}_{2}$ & $2 \mu \mathrm{H}$ \\
\hline
\end{tabular}

\subsection{Matching Network and Harmonic Trap}

A Smith chart was used to design the standard low pass Pi-section matching work that will transform the load impedance $Z_{L}=50 \Omega$ into $R=65 \Omega$ (see Section 4.2) at the impedance inverter output. With the exception of the capacitor that will be substituted for the second harmonic trap, all capacitors in the network are standard values by design. Tunable inductors were used to provide flexibility and to account for any extra tolerance, etc. that may have occurred in the capacitors. 
Table 4.2: Impedance and Q-factor at each labeled node in Figure 4.5 and Figure 4.6 .

\begin{tabular}{|c|c|c|}
\hline Node & Impedance & $\mathbf{Q}$ \\
\hline 1 & 50 & 0 \\
\hline 2 & $50-\mathrm{j} 1.6$ & 0 \\
\hline 3 & $11.9-\mathrm{j} 21.3$ & 1.8 \\
\hline 4 & $11.9+\mathrm{j} 25.8$ & 2.2 \\
\hline 5 & $68+\mathrm{j} 1.0$ & 0 \\
\hline
\end{tabular}

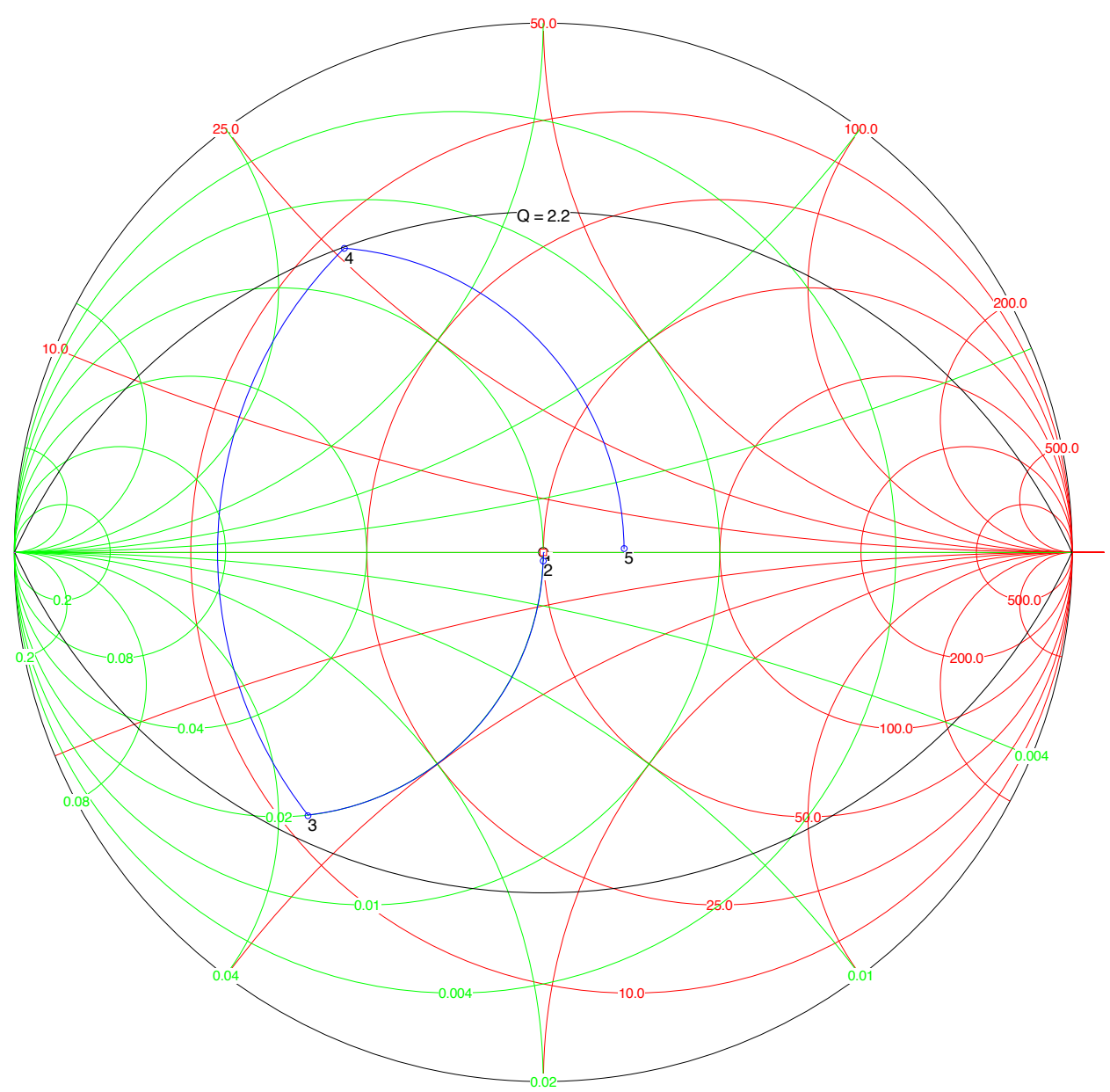

Figure 4.5: Smith chart design for "base"-topology matching network 


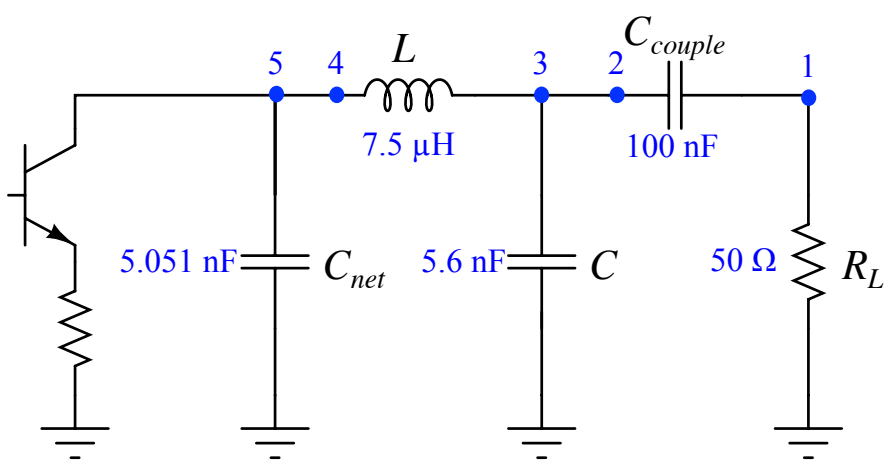

Figure 4.6: Matching network with resulting component values and node annotations.

A slight mismatch exists due to restricting the design to only standard value capacitors, but it is not significant enough-especially at the low frequency of $1 \mathrm{MHz}$ - to cause reflections or otherwise hinder the operation of the amplifier.

Using (3.10) and 3.11) yields a series LC second harmonic trap that can be substituted for capacitor $C_{\text {net }}$ :

$$
\begin{aligned}
X_{\mathrm{net}} & =-\frac{1}{\omega C_{\mathrm{net}}}=-31.5 \Omega \\
C_{s} & =-\frac{3}{4 \omega_{0} X_{\text {net }}} \approx 3.9 \mathrm{nF} \\
L_{s} & =\frac{1}{4 \omega_{0} C_{s}} \approx 1.55 \mu \mathrm{H}
\end{aligned}
$$

The completed matching network with harmonic trap is shown in Figure 4.7

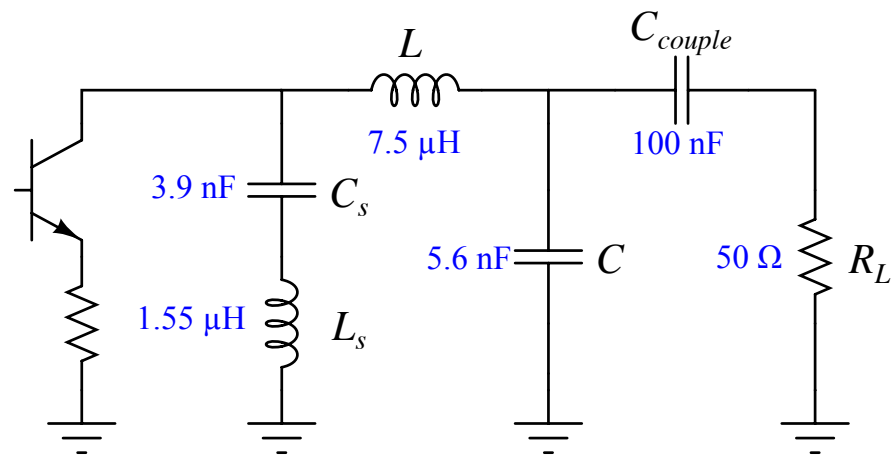

Figure 4.7: Completed matching network with harmonic trap. 


\subsection{Class C Feedback}

It was found from (3.6), implemented in the ideal equation-based driving source during simulation (see Section 4.3), that the peak magnitude at the base of the Class $\mathrm{C}$ should be $625 \mathrm{mV}$. Substituting this value into (3.12) and solving for $L_{\text {scale }}$ results in a value of approximately $2 \mu \mathrm{H}$ for the inverting transformer. The feedback configuration with calculated component values is given in Figure 4.8 and the resulting Class $\mathrm{C}$ stage operation (for swept values of input voltage) when the feedback is connected is displayed in Figure 4.9

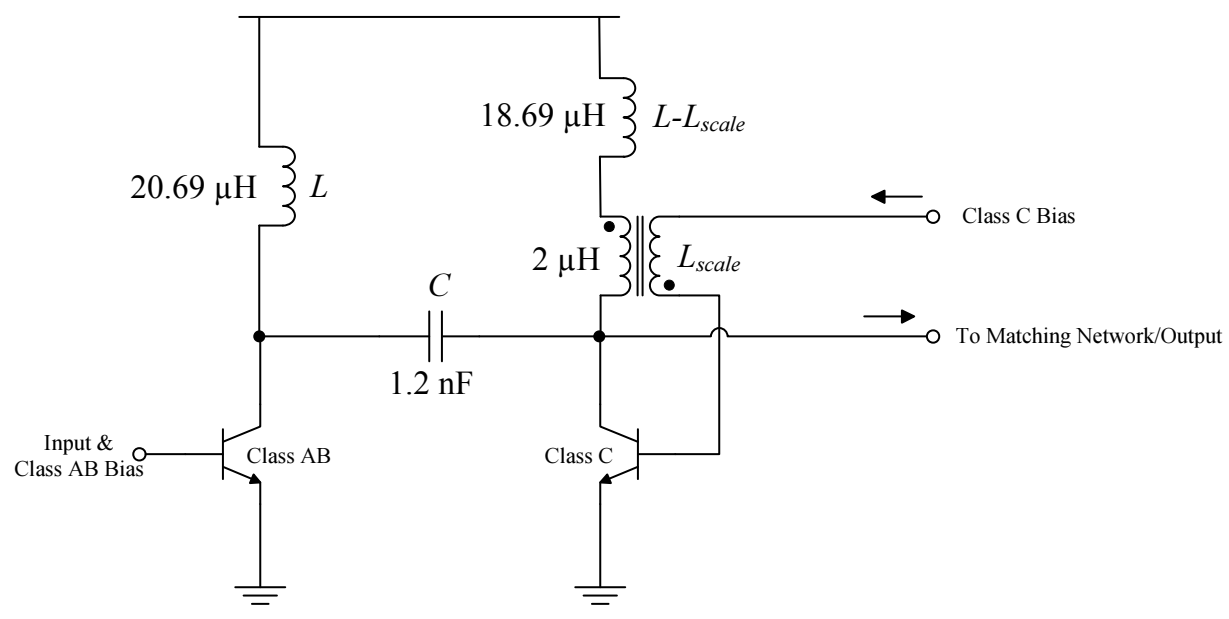

Figure 4.8: Class $\mathrm{C}$ partial positive feedback configuration with calculated component values.
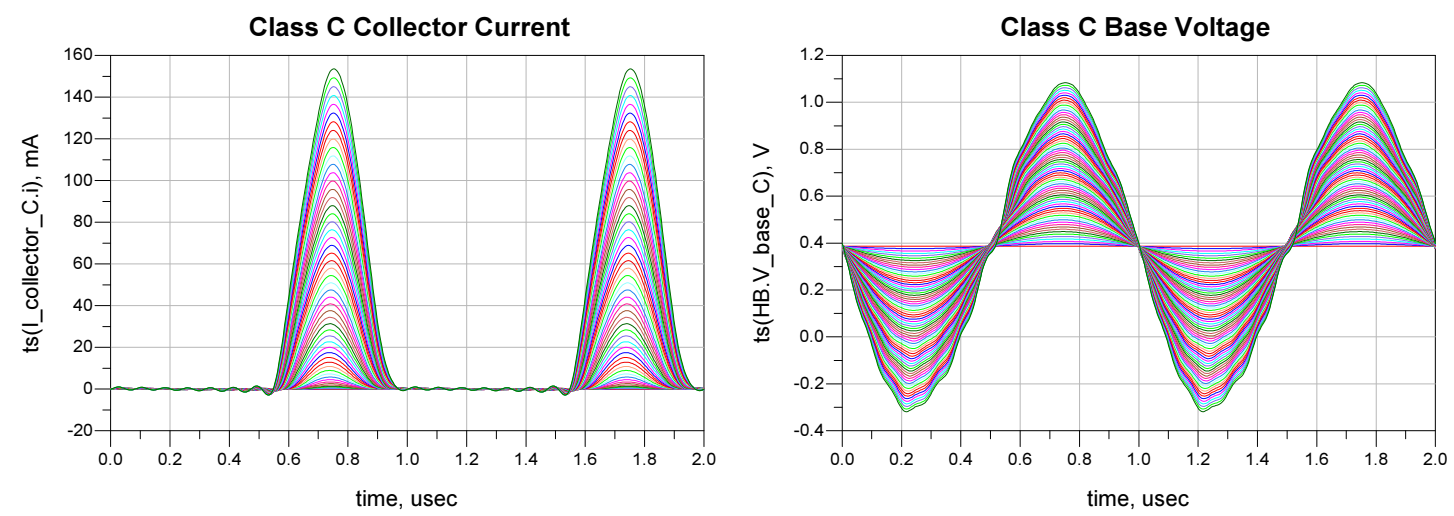

Figure 4.9: Class $C$ collector current and base voltage. 


\subsection{Completed Amplifier}

Agilent ADS simulation schematics for the fully-configured amplifier can be found in Appendix A The magnitudes of the Class $\mathrm{AB}$ and Class $\mathrm{C}$ fundamental collector voltages and currents over the full range of input voltages are shown in Figure 4.10 and Figure 4.11 . The peak amplitudes over the same range of input voltages are shown in Figure 4.12 .

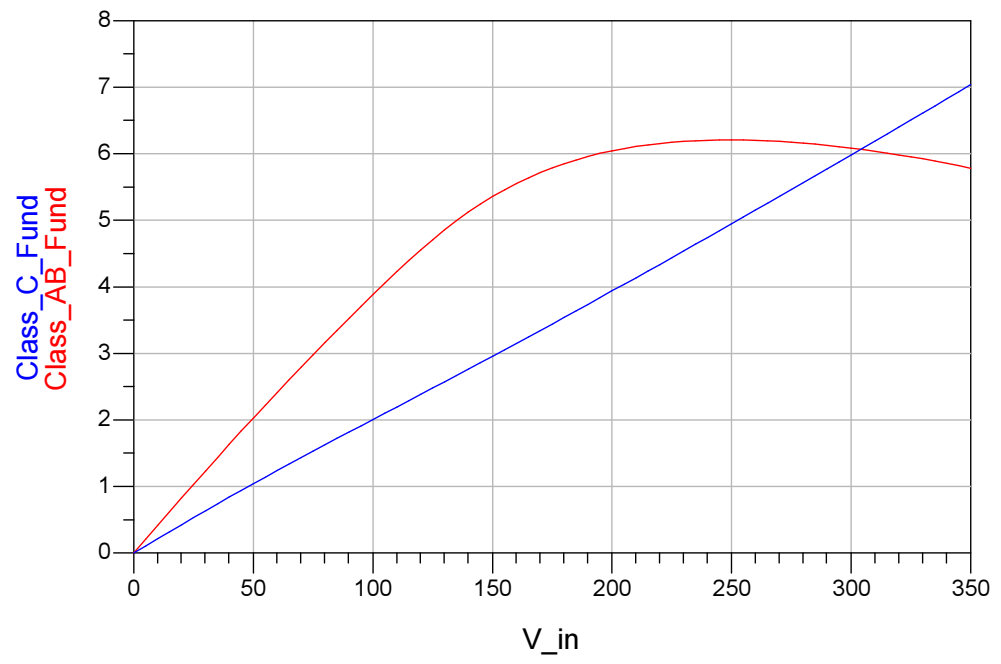

Figure 4.10: Magnitudes of Class $\mathrm{AB}$ and Class $\mathrm{C}$ collector voltage (fundamental components) vs. input voltage.

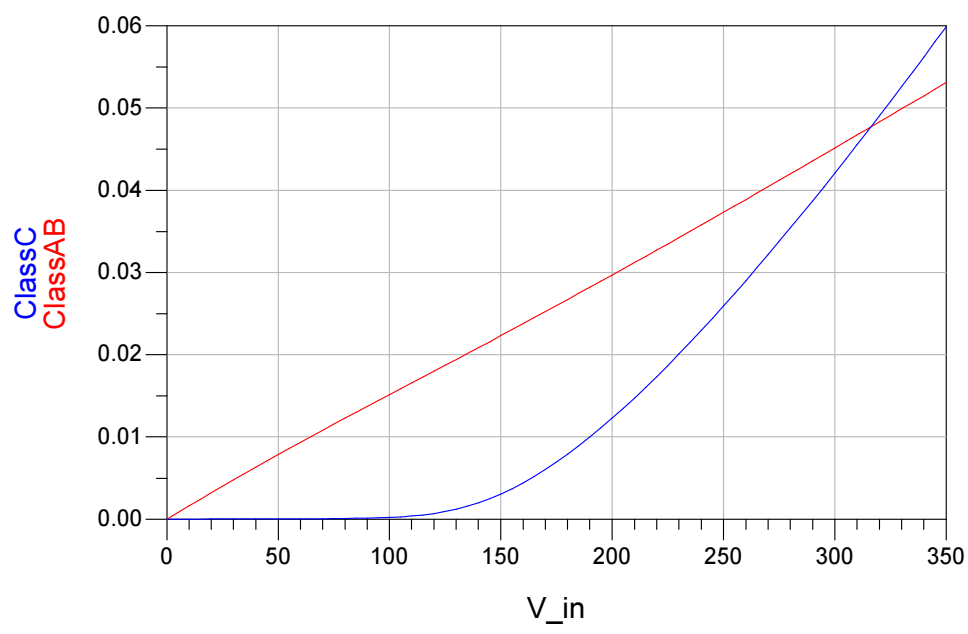

Figure 4.11: Magnitudes of Class AB and Class $C$ collector current (fundamental components) vs. input voltage. 
From Figure 4.10 and Figure 4.11, we see that the Class $\mathrm{C}$ stage activates more quickly than in the idealized simulation of Figure 4.4, and therefore reaches a higher maximum current than the Class $\mathrm{AB}$ stage. This has the effect of decreasing the fundamental magnitude of the Class $\mathrm{AB}$ voltage slightly at maximum drive levels. This discrepancy is caused by the voltage division ratio at the output of the impedance inverter: recall from Section 4.5 that the calculated value for $L_{\text {scale }}$ was subtracted from the series inductance $L$, so that their series combination added up to a total inductance of $L$. While this is necessary for proper functionality of the impedance inverter, it also alters the voltage division ratio that was used to determine the value of $L_{\text {scale }}$ originally, therefore resulting in a higher voltage magnitude being fed back onto the base of the Class $\mathrm{C}$ device and causing it to supply more current at maximum drive than intended. While this could be easily remedied by further optimization of the voltage division ratio, it illustrates another possible design case in that it may be desirable for the amplifier to have maximum efficiency at reduced drive levels in some situations-for example, in digital modulation schemes, there may be a certain range of signal amplitudes that are statistically more likely to occur than others, and it therefore may be useful to tune the amplifier to have maximum efficiency for this range of input amplitudes.

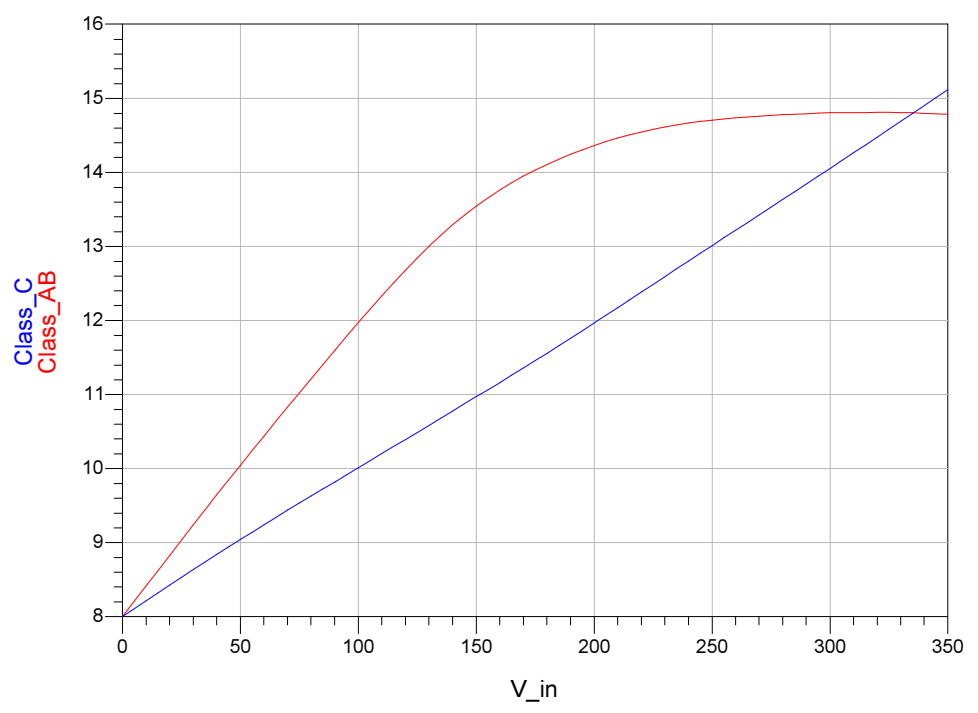

Figure 4.12: Peak amplitudes of Class $A B$ and Class $C$ collector voltage vs. input voltage. 
The peak amplitude of the output load voltage over the swept range of input values is shown in Figure 4.13 , confirming the linearity of the amplifier.

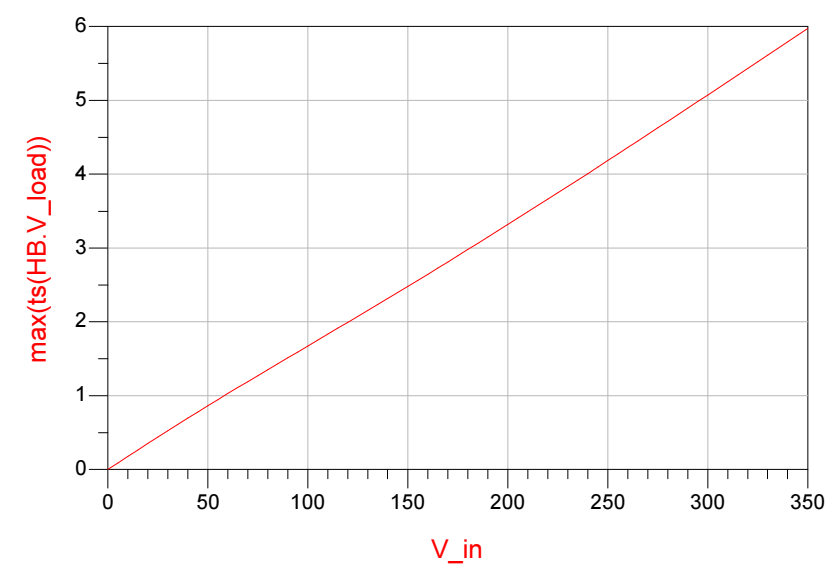

Figure 4.13: Peak amplitudes of output voltage vs. input voltage.

The power efficiency curve in Figure 4.14 shows a maximum efficiency of $63 \%$ at maximum input drive. Notice from the curve that if the designer began to back off on the gain of the amplifier, the efficiency degradation would be minor.

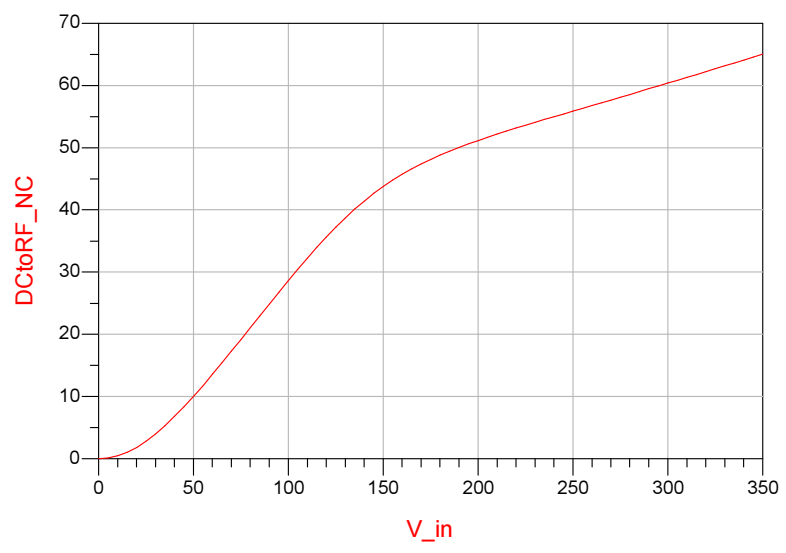

Figure 4.14: Power efficiency vs. input voltage.

\subsection{PCB Layout}

The completed amplifier was implemented on a two-layer FR-4 board using the Cadsoft EAGLE layout tools. The layout is shown in Figure 4.15, with some of the key sections labeled. Note that 
all traces shown in red occur on the top layer of the PCB, and those shown in blue occur on the bottom layer. To maintain an uncluttered layout, all Class $\mathrm{AB}$ and Class $\mathrm{C}$ collector traces were routed on the top layer, and all base traces were routed on the bottom layer. Further detail showing screenshots of the individual layers, etc. can be found in Appendix B

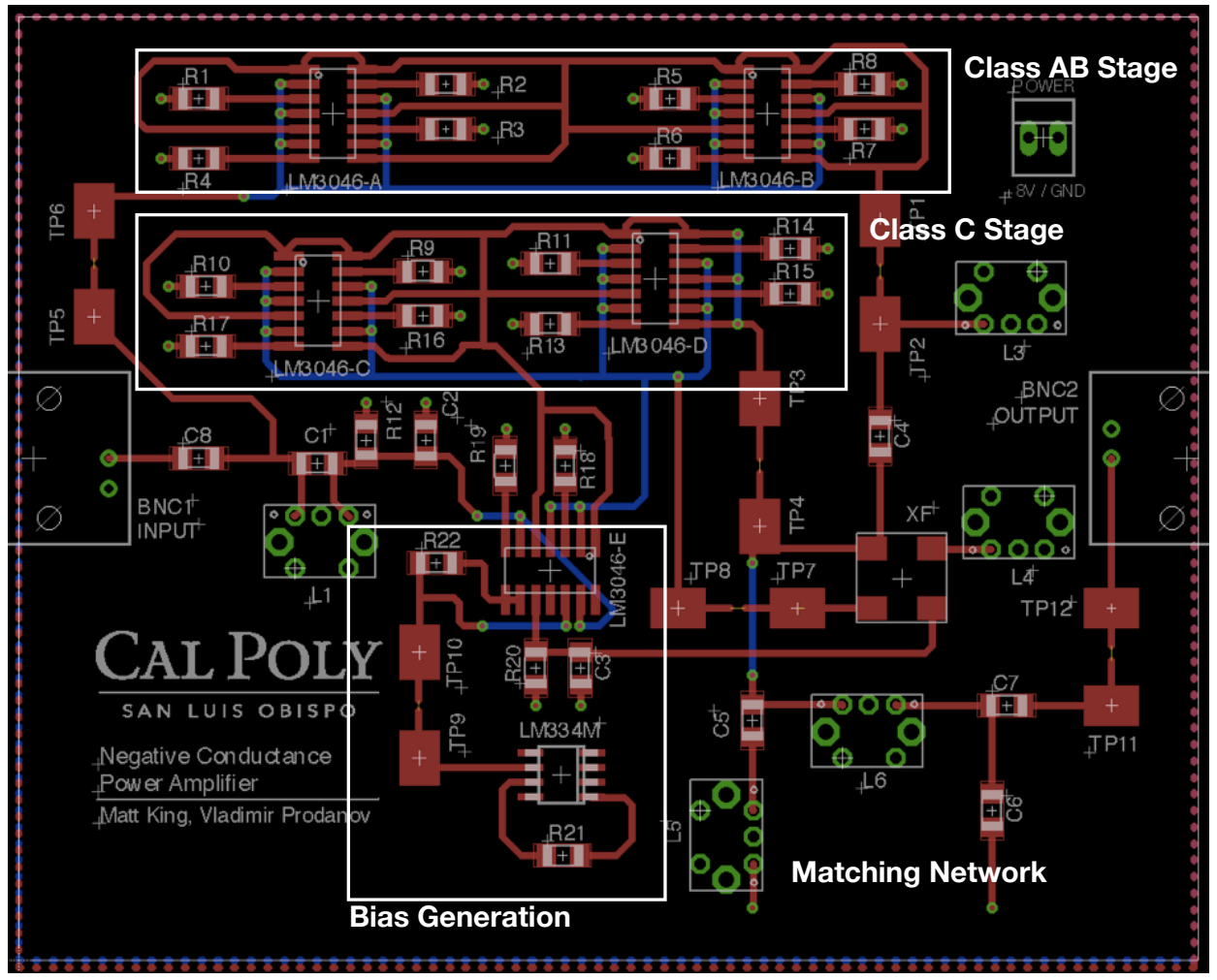

Figure 4.15: PCB layout of negative conductance amplifier. 


\section{Chapter 5}

\section{Prototype Test Results}

Due to issues with the PCB prototype, a second prototype (with same layout and components) had to be fashioned on a solderable breadboard. Test results for both prototypes are presented.

\subsection{Prototype $1(\mathrm{PCB})$}

The completed PCB prototype is shown in Figure 5.1

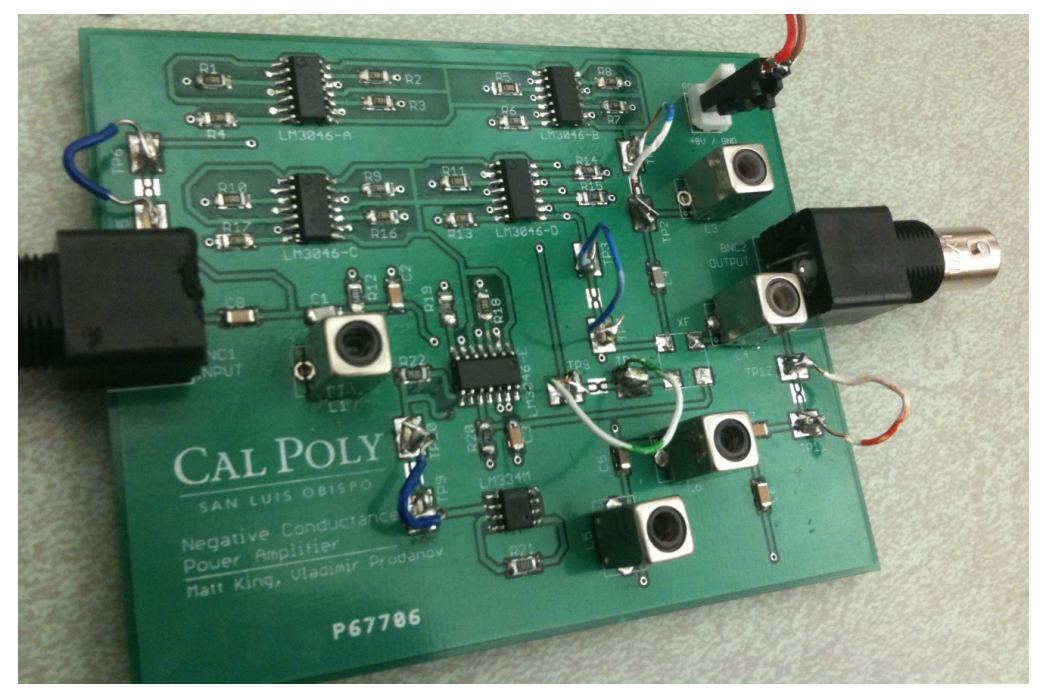

Figure 5.1: Completed PCB prototype. 
Initially, the Class $\mathrm{C}$ amplifier was left disconnected, and the Class $\mathrm{AB}$ amplifier was connected and driven with a sinusoidal input signal to observe the proper operation of the impedance inverter and matching network. Figure 5.2 shows the voltages developed at the impedance inverter input (labeled 'Class $\mathrm{AB}$ ') and output (labeled 'Class C') for a $530 \mathrm{mV}$ pp input signal.

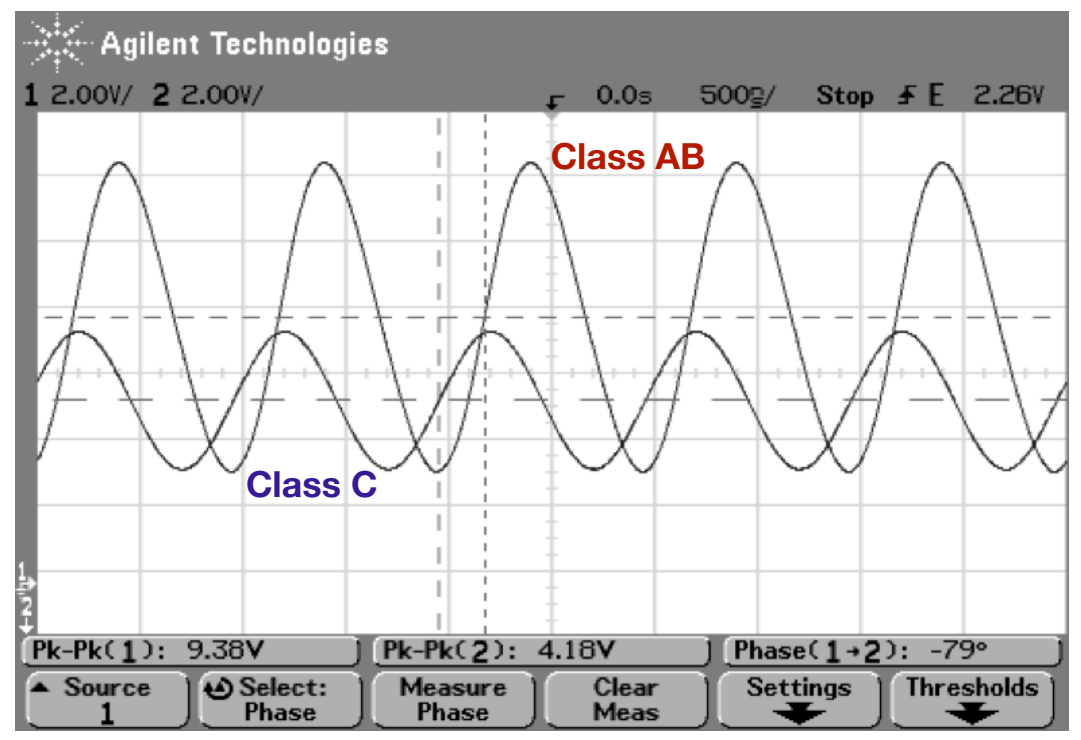

Figure 5.2: Voltage waveforms at impedance inverter input (Class $A B$ side) and output (Class $\mathrm{C}$ side).

The voltage amplitudes observed at the input and the output are consistent with theory according to (2.2) and (2.3). Recall from the design in Section 4.2 that $X=130 \Omega$ and $R=65 \Omega$. and Since the Class $\mathrm{C}$ stage was disconnected, $I_{2}=0$ and the following should be true:

$$
\begin{array}{r}
V_{1}=\frac{X^{2}}{R} I_{1}+j X I_{2}=\frac{130^{2}}{65} I_{1}=260 I_{1} \\
V_{2}=j X I_{1}=j 130 I_{1} \\
\frac{V_{2}}{V_{1}}=\frac{j 130 I_{1}}{260 I_{1}}=\frac{j}{2} \\
V_{2}=\frac{j}{2} I_{1}
\end{array}
$$

The impedance inverter output amplitude should be half of the input amplitude, and shifted by $90^{\circ}$ with respect to the input. A comparison of the theoretical voltages to the measured voltages for an impedance inverter input of $9.38 \mathrm{~V}_{\mathrm{pp}}$ is given in Table 5.1 
Table 5.1: Comparison of theoretical and measured impedance inverter voltages.

\begin{tabular}{|r|c|c|}
\hline & Theoretical & Measured \\
\hline Input Voltage & $9.38 \mathrm{Vpp}$ & $9.38 \mathrm{Vpp}$ \\
\hline Output Voltage & $4.69 \mathrm{Vpp}$ & $4.18 \mathrm{Vpp}$ \\
\hline Voltage Ratio & 2 & 2.2 \\
\hline Phase Shift & $90^{\circ}$ & $79^{\circ}$ \\
\hline
\end{tabular}

Next, the output of the impedance inverter was connected to the base of the Class $\mathrm{C}$ stage, and the Class $\mathrm{C}$ collector was connected to the supply rail, in order to verify proper attenuation and inversion of the signal being fed back on to the Class $\mathrm{C}$ base. The measured impedance inverter output and Class $\mathrm{C}$ base voltage are shown in Figure 5.3, showing that the base voltage is indeed attenuated by a factor of 10 , and inverted as desired.

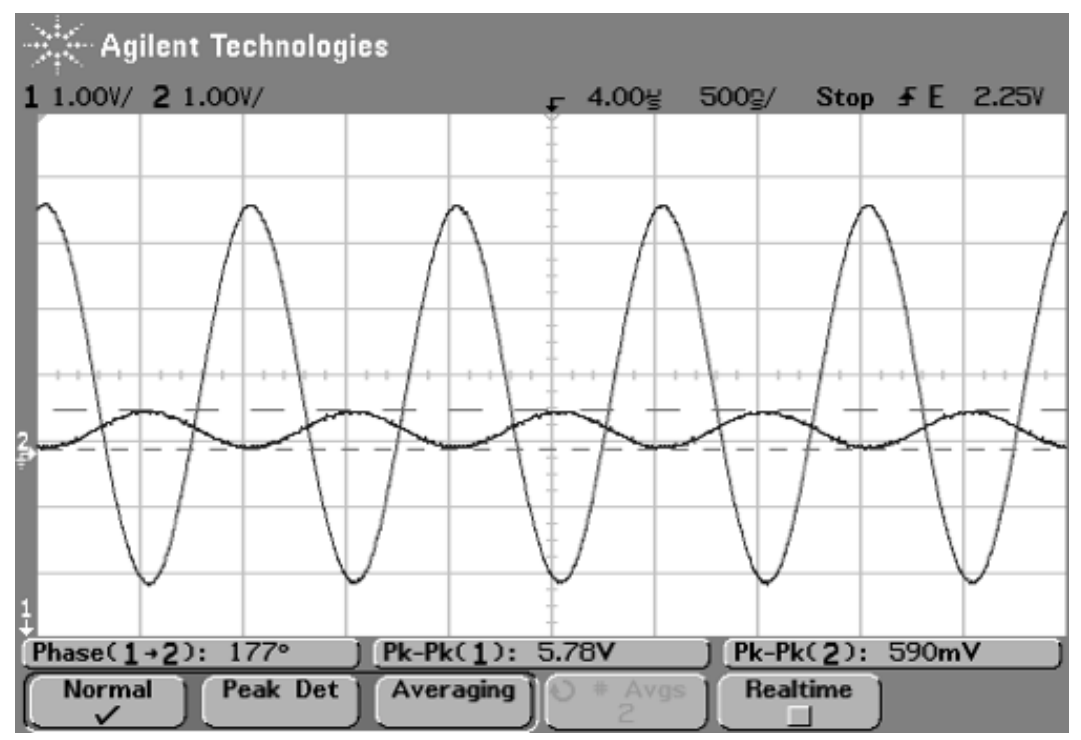

Figure 5.3: Impedance inverter output voltage and Class $C$ base voltage, verifying proper attenuation and inversion of the feedback signal.

A $1 \mathrm{k} \Omega$ resistor was placed between the supply rail and the Class $C$ collector to verify that the collector voltage displayed a reduced conduction-angle characteristic indicative of proper Class $\mathrm{C}$ operation (see Figure 5.4). 


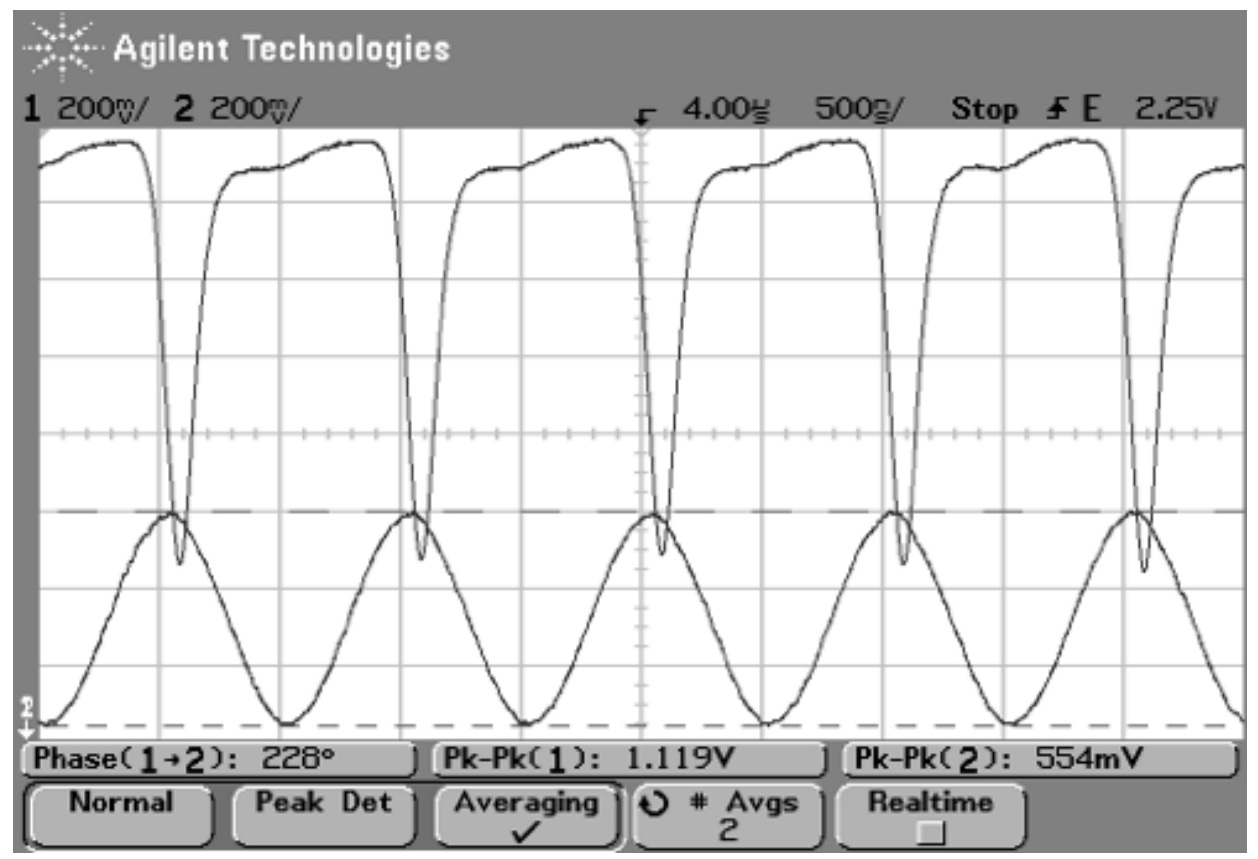

Figure 5.4: Class $C$ collector voltage and base voltage.

The operation of the complete amplifier was unable to be verified, due to certain phenomena that occurred once the Class $\mathrm{C}$ collector was connected to the impedance inverter output (thereby completing the feedback). In this configuration, whenever measurements were attempted at the collector nodes with oscilloscope probes, the DC power supply would rapidly reach its set current compliance level and cut power to the circuit. This was indicative of some sort of a fast, largeamplitude transient that occurred when the circuit was loaded with the scope probe. In order to diagnose this issue, a replica of the PCB prototype was constructed on a solderable breadboard so that circuit modifications could be more easily implemented.

\subsection{Prototype 2 (Solderable Breadboard)}

The completed second prototype on a solderable breadboard is shown in Figure 5.5. Initially, the second prototype exhibited the same issue when the collector nodes were probed. Surface mount resistors were then placed in parallel with the inductors in the impedance inverter in order to reduce their Q, and hence, any peaking that may have been occurring when the scope probe was applied to 
the circuit. This alleviated the issue, and further measurements were able to be completed.

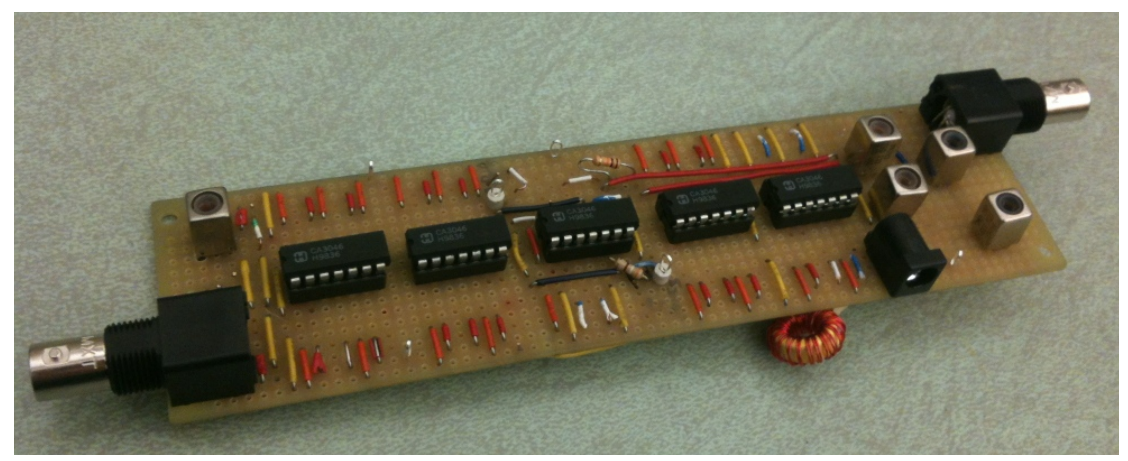

Figure 5.5: Completed prototype on solderable breadboard.

The measured DC bias levels for the Class $\mathrm{AB}$ and Class $\mathrm{C}$ stages are shown in Table 5.2. Notice that the bias levels are higher than the designed values; this resulted in the first stage to operate in a Class A mode (i.e., conducting for the entire cycle), as opposed to Class AB.

Table 5.2: Comparison of simulated and measured DC bias levels.

\begin{tabular}{|r|c|c|}
\hline & Simulated & Measured \\
\hline Class AB & $669 \mathrm{mV}$ & $749 \mathrm{mV}$ \\
\hline Class C & $400 \mathrm{mV}$ & $450 \mathrm{mV}$ \\
\hline
\end{tabular}




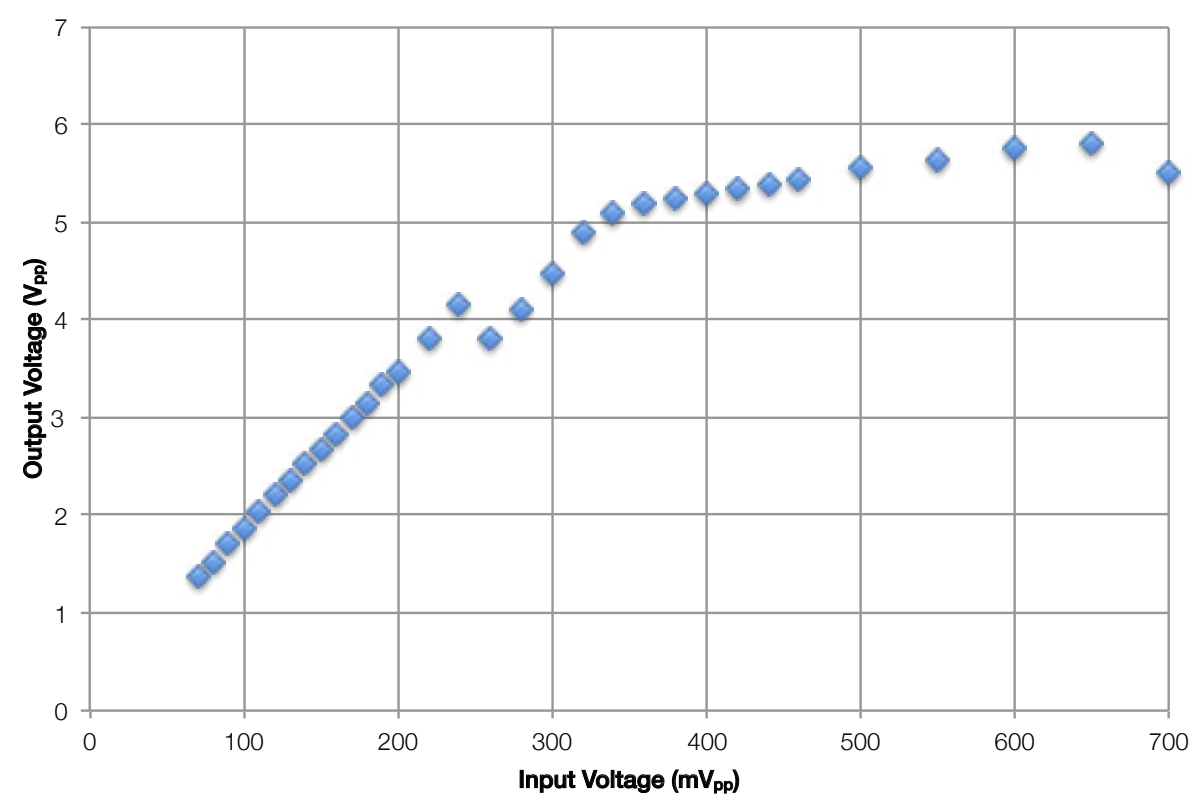

Figure 5.6: Measured output voltages (peak-to-peak values).

A plot of measured output voltages is given in Figure 5.6, and the measured Class AB and Class $\mathrm{C}$ collector voltages are shown in Figure 5.10. Measurements were difficult to obtain at drive levels above $300 \mathrm{mV}_{\mathrm{pp}}$ due to noise or other fluctuations.

The frequency spectra at the amplifier output for various input drive levels are shown in Figures 5.7. 5.8, and 5.9. In Figure 5.7, for an input drive of $90 \mathrm{mV}_{\mathrm{pp}}$, the Class $\mathrm{C}$ device has not yet been activated. The frequency spectrum shows the fundamental component and an attenuated second harmonic component, verifying that the integrated second harmonic trap in the matching network was functional. At an input drive of $190 \mathrm{mV}_{\mathrm{pp}}$, the Class $\mathrm{C}$ had begun to activate, indicated by the presence of higher-order harmonics shown in Figure 5.8. For an input drive of $210 \mathrm{mV}_{\mathrm{pp}}$ (Figure 5.9), the Class $\mathrm{C}$ device was on and injecting current with high harmonic content into the impedance inverter. The resulting distorted voltage was fed back onto the base, which accounts for the significant higher-order harmonic content. 


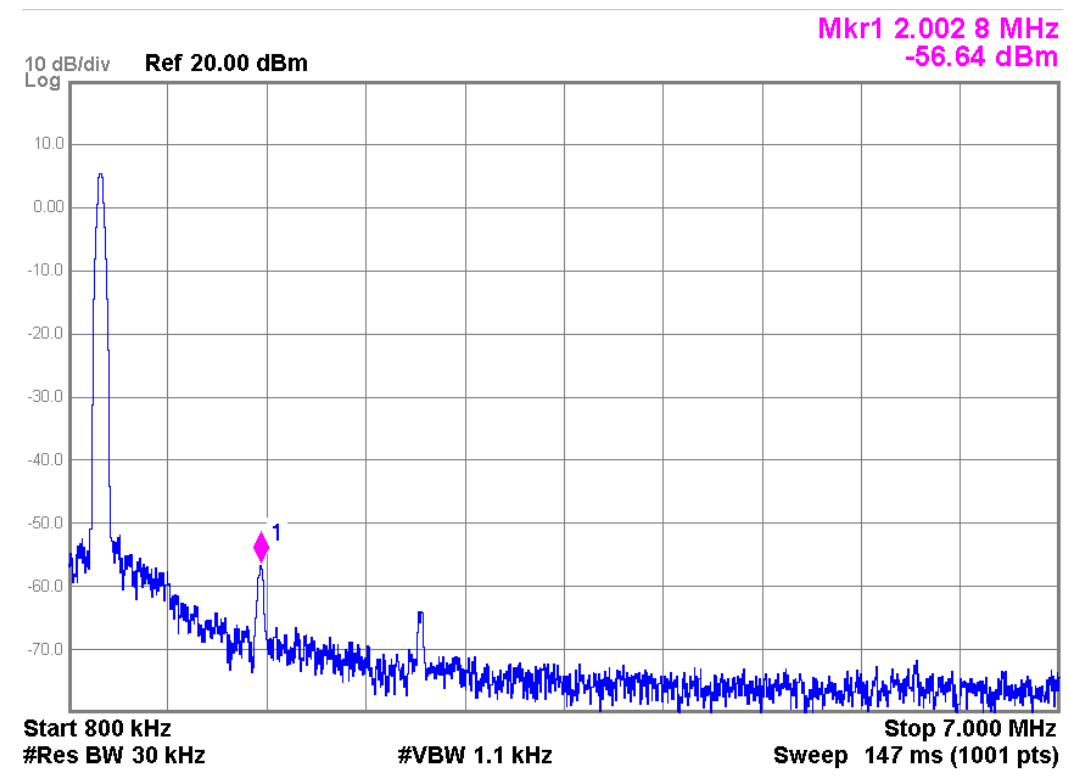

Figure 5.7: Output frequency spectrum for input drive of $90 \mathrm{mV}$ pp.

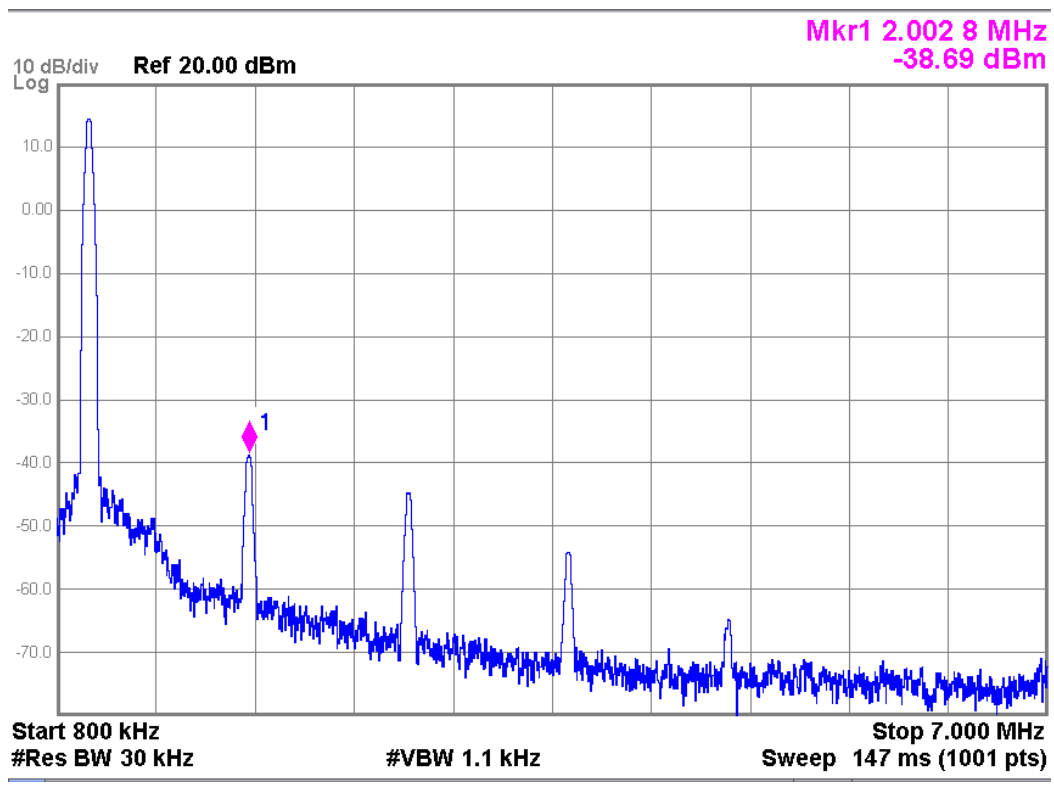

Figure 5.8: Output frequency spectrum for input drive of $190 \mathrm{mV}$ p. 


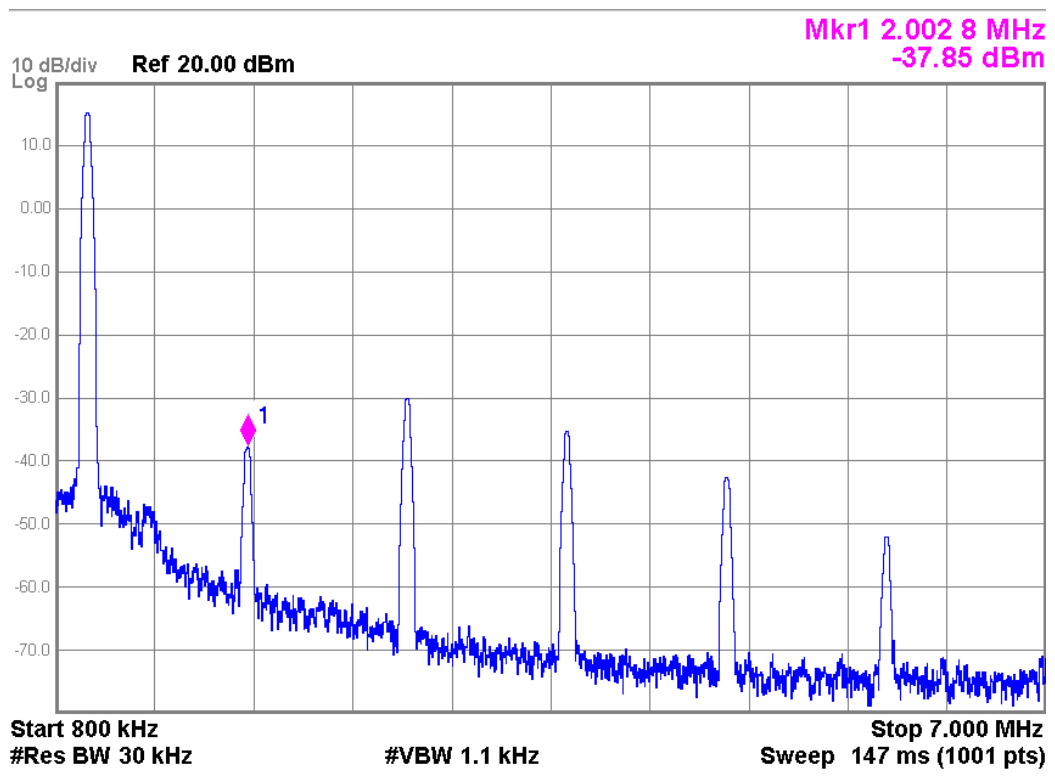

Figure 5.9: Output frequency spectrum for input drive of $210 \mathrm{mV}_{\mathrm{pp}}$.

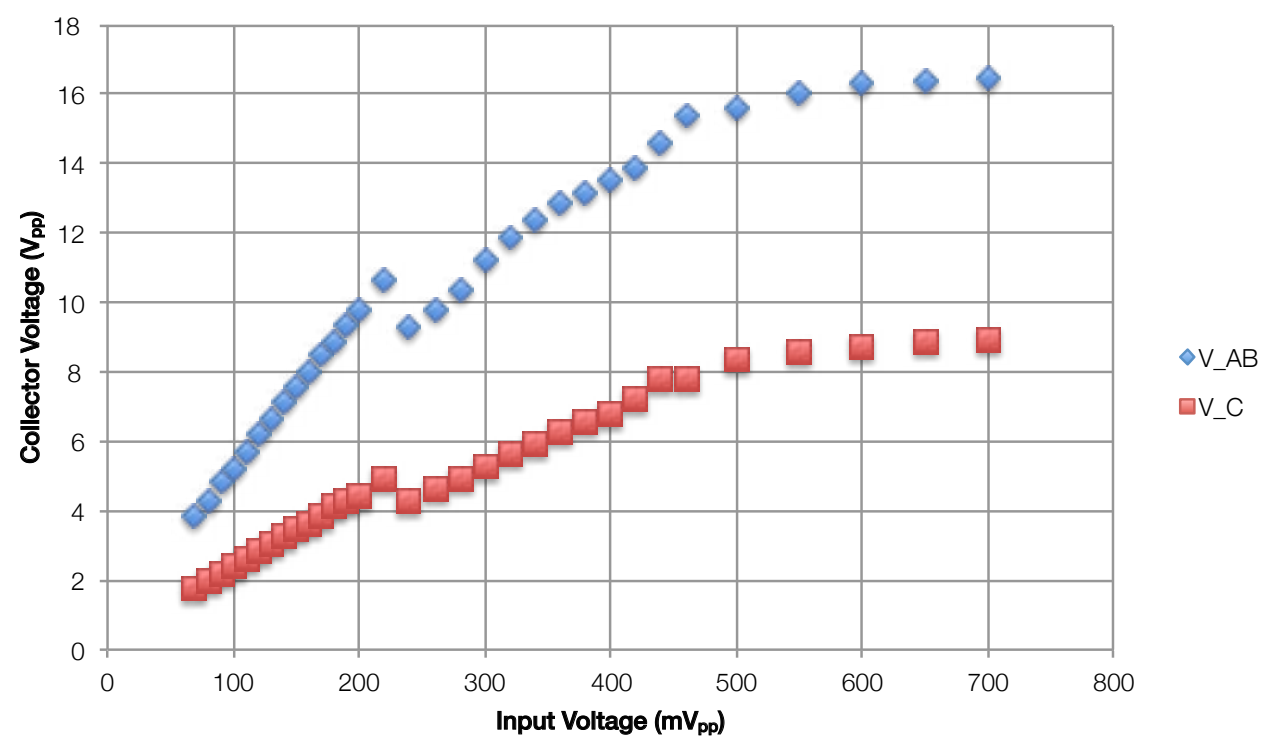

Figure 5.10: Measured Class $\mathrm{AB}$ and Class $\mathrm{C}$ collector voltages (peak-to-peak values).

The power efficiencies over the range of input voltages are plotted in Figure 5.11. These were calculated from the measured RF power (using the RMS voltage at the output $50 \Omega$ load) and the DC power from the supply. The maximum efficiency was $25.37 \%$ for a $350 \mathrm{mV}_{\mathrm{pp}}$ input, and the efficiency at maximum input drive was $21.74 \%$. 


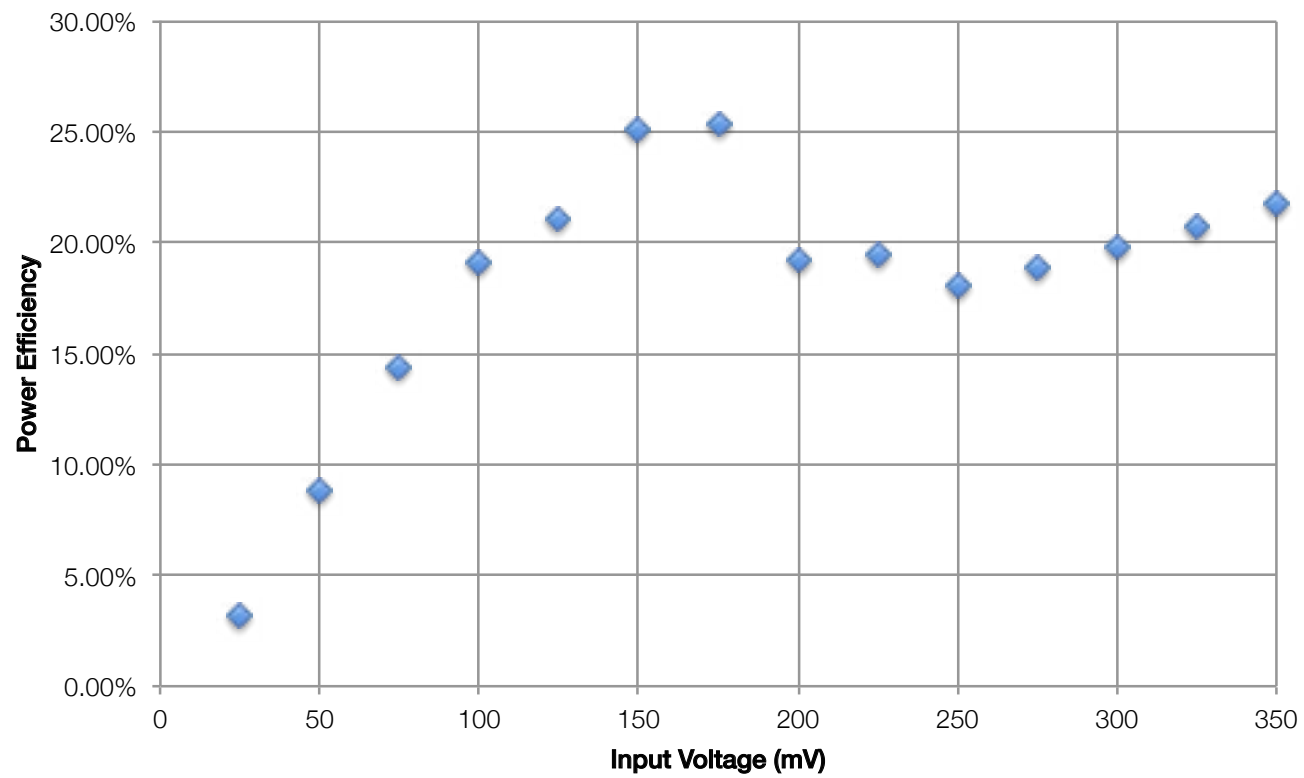

Figure 5.11: Plot of measured power efficiencies. 


\section{Chapter 6}

\section{Conclusions and Future Work}

From the results obtained for Prototype 1 in Section 5.1, it is clear that the negative conductance topology itself is valid and worthy of further research. The partial positive feedback mechanism worked as intended, and the lumped element impedance inverter provided the required $90^{\circ}$ phase shift to achieve the Doherty effect. From the results in Figures 5.6 and 5.10 for Prototype 2, Doherty action was clearly observed since the rate of growth of the output signal magnitude began to decrease as the Class $\mathrm{C}$ negative conductance stage activated and began to supply current. However, the presence of distortion on the output for increased drive levels suggests that harmonic content generated by the Class $\mathrm{C}$ device may have been fed back to the base of the Class $\mathrm{C}$ device, in addition to the fundamental component. Therefore, it may be effective to apply low-pass filtering to the feedback loop as a safeguard against amplification of any higher-order harmonic content that is not suppressed by the harmonic trap.

The measured efficiency of the amplifier follows the general trend of the simulated efficiency curve, in that the efficiency at high drive levels is not reduced by any significant amount if the designer were to begin backing off on the amplifier gain. However, the overall measured efficiency of the amplifier was significantly lower than in simulation, for multiple reasons:

- Resistors of relatively large value — on the order of $100 \Omega$ - were added to the circuit to "deQ" the impedance inverter inductors (see Section 5.2). This results in an significant amount 
of power being dissipated in the resistor, which greatly reduces the power efficiency of the amplifier since this dissipated power is not being supplied to the load.

- As mentioned previously, harmonic content was likely being fed back to the base of the Class $\mathrm{C}$ once the Class $\mathrm{C}$ device activated, which resulted in the higher-order harmonics being amplified. This has the effect of reducing the fundamental component of the power delivered to the load, which also lowers the efficiency of the amplifier.

- The DC bias levels for both stages turned out to be higher than their target values. In the case of the input stage, the bias was high enough to place the amplifier in a Class A mode rather than Class $\mathrm{AB}$, thereby lowering the efficiency of the amplifier since the input stage was activated and conducting during the full cycle of the input signal.

Even with the reduction in efficiency, the maximum power efficiency of the tested prototype still exceeds that of some commercially-available mobile power amplifiers, which have efficiencies as low as $12 \%[7]$.

The LM3046 devices themselves were also a major contributor to the non-idealities observed during testing, due to the very low operating frequency that was chosen for the prototype design. The LM3046 is rated for operation from DC to $120 \mathrm{MHz}$; choosing the operating frequency to be $1 \mathrm{MHz}$ meant that the higher-order harmonics relative to the operating frequency (i.e. $3 \mathrm{MHz}, 5$ $\mathrm{MHz}, 7 \mathrm{MHz}$, etc.) were well within the bandwidth of these transistors and therefore sustained in the system. Therefore, while a low operating frequency is useful for simplifying the amplifier layout, caution must be exercised to ensure that the frequency is not low enough that unwanted harmonics are enhanced by the devices themselves. If the prototype is redesigned to operate at 20 $\mathrm{MHz}$, for example, any harmonics of order $6(120 \mathrm{MHz})$ or higher will not be enhanced by the LM3046. It is likely that the higher-order harmonic enhancement mentioned above was partly at fault for the issue presented in Section 5.1, in which high-frequency transients caused by scope probe loading may have been fed back to the Class $\mathrm{C}$ input and subsequently amplified.

The multi-fingered approach used in this design, while common for IC applications, may not be suitable for PCB implementations using discrete transistors or transistor arrays. Integrated NPN 
BJT devices connected in parallel, when saturated, can exhibit unwanted behavior such as substrate de-biasing and current hogging, in which one transistor in the parallel combination may demand more base current at the expense of the other transistors connected in parallel [5] $]^{1}$ A common protection method against these phenomena is to connected a Schottky diode between the collector and base terminals of the combined devices [5], as shown in Figure 6.1] thereby "clamping" the collector-base PN junction at a low voltage that prevents it from saturating.

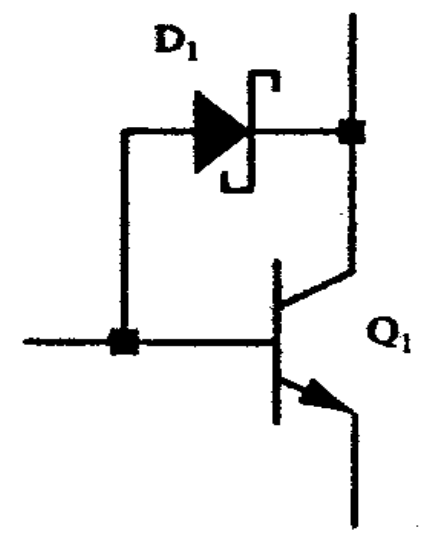

Figure 6.1: Schottky-clamped NPN transistor [5].

A future implementation of this design is planned, which will employ single-transistor amplifying stages [in contrast to the multi-fingered approach outlined in this work] with separate biasing for each stage ${ }^{2}$ The new implementation will also be designed for slightly higher-frequency operation $(10-20 \mathrm{MHz})$, with the goal that higher-order harmonics will no longer be enhanced by the transistors. This should eliminate the need for Q-reducing resistors that will degrade the efficiency of the amplifier. An input matching network for a $50 \Omega$ driving source will also be included in the next prototype; a well-defined impedance presented to the amplifier input will allow for the power gain of the amplifier to also be well-defined. Two-tone intermodulation tests will also be performed, since these tests could not be performed within the time frame of the current work. The results of this upcoming prototype will be presented in a later publication.

\footnotetext{
${ }^{1}$ Having devices on two different chips, and therefore two different substrates, connected in parallel to form a single amplifying device may have complicated this issue even further.

${ }^{2}$ The multi-output bias network proposed in this work may not be a viable option, as evidenced by the higher-thanexpected DC bias levels measured in Section 5.2
} 


\section{Appendix A}

\section{Circuit Schematics}

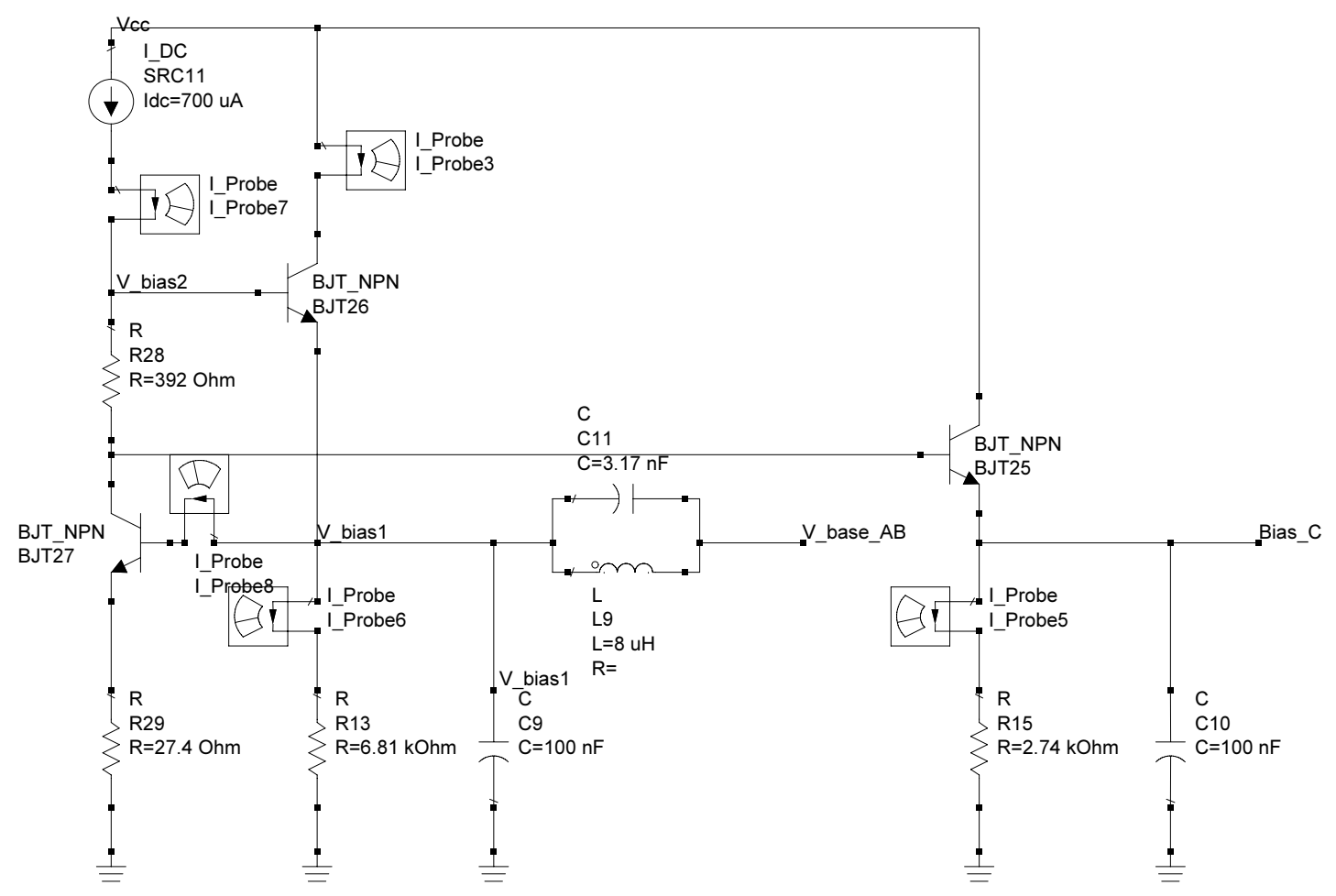

Figure A.1: Bias circuit. Nodes $V \_b a s e \_A B$ and Bias $_{-} C$ are the connected to the bases of the Class $\mathrm{AB}$ and Class $\mathrm{C}$ stages, respectively. 


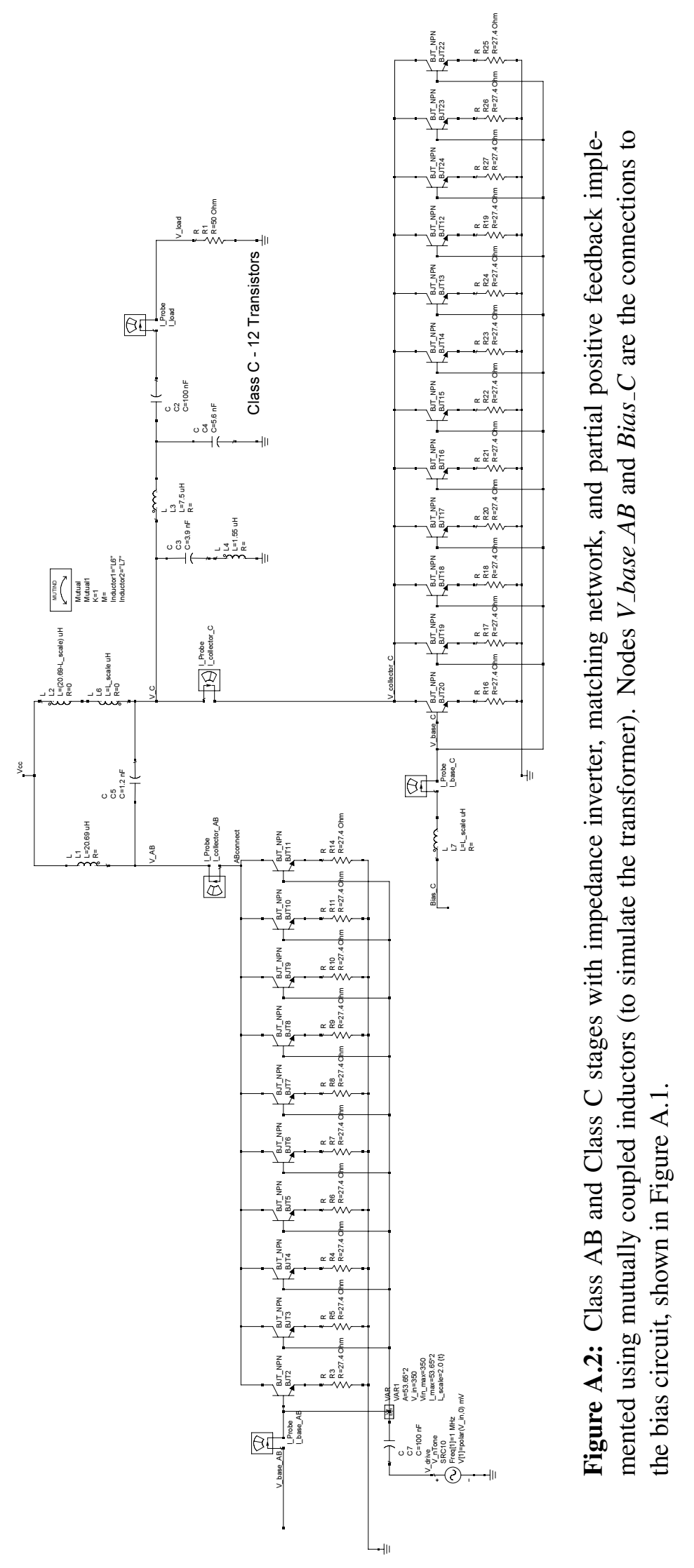




\section{Appendix B}

\section{PCB Layout Detail}

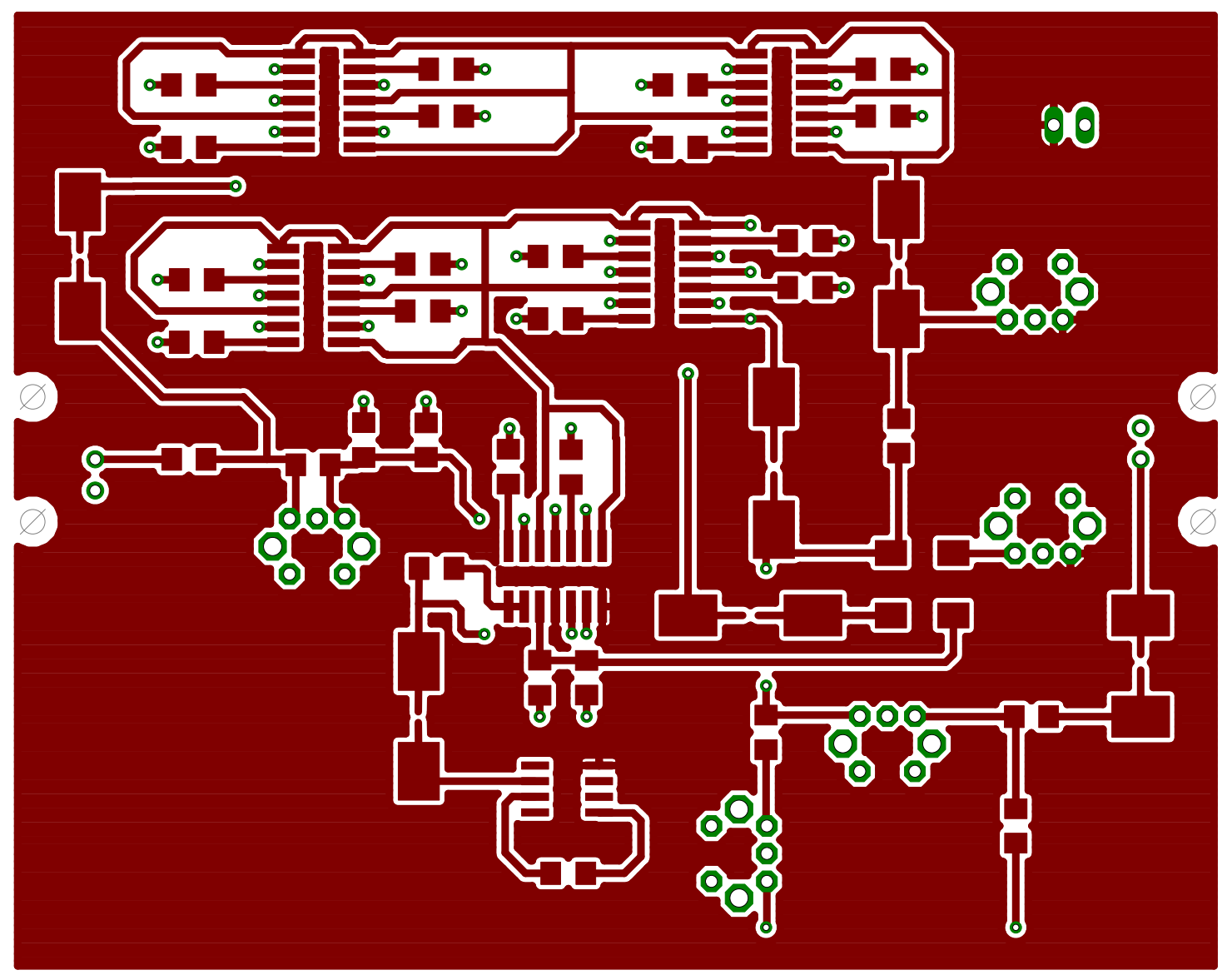

Figure B.1: View of PCB top layer. All collector traces are routed on this layer, and a power supply plane covers the unused area. 


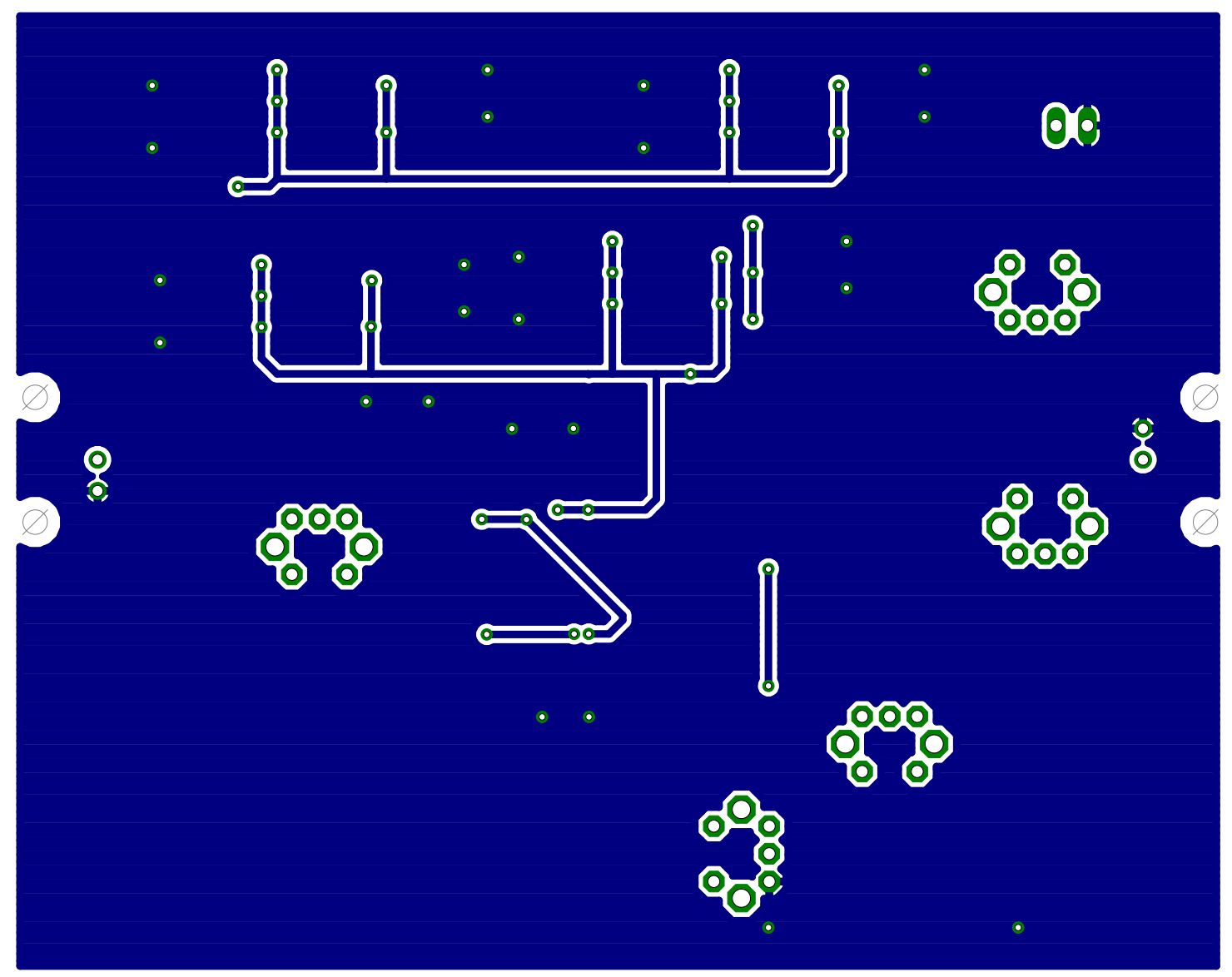

Figure B.2: View of PCB bottom layer. All base traces are routed on this layer, and a ground plane covers the unused area. 


\section{References}

[1] S. C. Cripps. RF Power Amplifiers for Wireless Communications. 2nd. Norwood, MA: Artech House, 2008.

[2] W. H. Doherty. "A new high efficiency power amplifier for modulated waves". In: Proceedings of the IRE. Vol. 24. Sept. 1936, pp. 1163-1182.

[3] D. W. Ferwalt. "A Base Control Doherty Power Amplifier Design for Improved Efficiency in GSM Handsets”. MS Thesis. Oregon State University, Dec. 2003.

[4] G. Gonzalez. Microwave Transistor Amplifiers. 2nd. Upper Saddle River, NJ: Prentice Hall, 1997.

[5] A. Hastings. The Art of Analog Layout. 1st. Upper Saddle River, NJ: Prentice Hall, 2001.

[6] B. Kim, J. Moon, and I. Kim. "Efficiently Amplified". In: IEEE Microwave Magazine (Aug. 2010).

[7] C. Neslen. "Negative Conductance Load Modulation Power Amplifier". MS Thesis. Cal Poly State University, June 2010.

[8] V. Prodanov. “Negative Conductance Power Amplifier”. Pat. 7,135,931 (US). 2006.

[9] V. Prodanov and M. Banu. "Power Amplifier Principles and Modern Design Techniques". In: Wireless Technologies: Circuits, Systems, and Devices. CRC Press, 2008. 